\title{
Extending tamely ramified strict 1-motives into két $\log$ 1-motives
}

\author{
Heer Zhao \\ Fakultät für Mathematik, Universität Duisburg-Essen, Essen 45117, Germany.
}

Received: 15 January 2020; Revised: 26 October 2020; Accepted: 25 December 2020

2020 Mathematics Subject Classification: Primary - 14D06; Secondary - 14A21, 14K99, 11G99

\begin{abstract}
We define két abelian schemes, két 1-motives and két log 1-motives and formulate duality theory for these objects. Then we show that tamely ramified strict 1-motives over a discrete valuation field can be extended uniquely to két $\log 1$-motives over the corresponding discrete valuation ring. As an application, we present a proof to a result of Kato stated in [12, §4.3] without proof. To a tamely ramified strict 1 -motive over a discrete valuation field, we associate a monodromy pairing and compare it with Raynaud's geometric monodromy.
\end{abstract}

\section{Notation and conventions}

Let $S$ be an fs log scheme. We denote by (fs $/ S$ ) the category of fs $\log$ schemes over $S$ and denote by $(\mathrm{fs} / S)_{\text {ét }}\left(\right.$ respectively $(\mathrm{fs} / S)_{\text {két }}$, respectively $(\mathrm{fs} / S)_{\mathrm{fl}}$, respectively $\left.(\mathrm{fs} / S)_{\mathrm{kfl}}\right)$ the classical étale site (respectively Kummer étale site, respectively classical flat site, respectively Kummer flat site) on (fs/S). In order to shorten formulas, we will mostly abbreviate (fs $/ S)_{\text {ét }}$ (respectively $(\mathrm{fs} / S)_{\text {két }}$, respectively $(\mathrm{fs} / S)_{\mathrm{fl}}$, respectively $\left.(\mathrm{fs} / S)_{\mathrm{kfl}}\right)$ as $S_{\text {ét }}$ (respectively $S_{\text {két }}$, respectively $S_{\mathrm{fl}}$, respectively $S_{\mathrm{kfl}}$ ). We refer to [7, §2.5] for the definitions of the classical étale site and the Kummer étale site, and [11, Def. 2.3] and $[15, \S 2.1]$ for that of the Kummer flat site. The definition of the classical flat site is an obvious analogue of that of the classical étale site. Then we have two natural 'forgetful' maps of sites:

$$
\varepsilon_{\text {ét }}:(\mathrm{fs} / S)_{\text {két }} \rightarrow(\mathrm{fs} / S)_{\text {ét }}
$$

and

$$
\varepsilon_{\mathrm{fl}}:(\mathrm{fs} / S)_{\mathrm{kfl}} \rightarrow(\mathrm{fs} / S)_{\mathrm{fl}}
$$

Kato's multiplicative group (or the log multiplicative group) $\mathbb{G}_{\mathrm{m}, \log }$ is the sheaf on $S_{\text {ét }}$ defined by $\mathbb{G}_{\mathrm{m}, \log }(U)=\Gamma\left(U, M_{U}^{\mathrm{gp}}\right)$ for any $U \in(\mathrm{fs} / S)$, where $M_{U}$ denotes the log structure of $U$ and $M_{U}^{\mathrm{gp}}$ denotes the group envelope of $M_{U}$. The Kummer étale sheaf $\mathbb{G}_{\mathrm{m}, \log }$ is also a sheaf on $S_{\mathrm{kfl}}$; see [15, Cor. 2.22] for a proof.

By convention, for any sheaf of abelian groups $F$ on $S_{\mathrm{kfl}}$ and a subgroup sheaf $G$ of $F$ on $S_{\mathrm{kfl}}$, we denote by $(F / G)_{S_{\text {et }}}$ (respectively $(F / G)_{S_{\text {fl }}}$, respectively $(F / G)_{S_{\text {két }}}$ ) the quotient sheaf on $S_{\text {ét }}$ (respectively $S_{\mathrm{fl}}$, respectively $S_{\text {két }}$ ), and $F / G$ denotes the quotient sheaf on $S_{\mathrm{kfl}}$. We abbreviate the quotient sheaf $\mathbb{G}_{\mathrm{m}, \log } / \mathbb{G}_{\mathrm{m}}$ on $S_{\mathrm{kfl}}$ as $\overline{\mathbb{G}}_{\mathrm{m}, \log }$.

(C) The Author(s), 2021. Published by Cambridge University Press. This is an Open Access article, distributed under the terms of the Creative Commons Attribution-NonCommercial-NoDerivatives licence (http://creativecommons.org/licenses/by/4.0/), which permits non-commercial reuse, distribution, and reproduction in any medium, provided the original work is unaltered and is properly cited. The written permission of Cambridge University Press must be obtained for commercial re-use or in order to create a derivative work. 


\section{Introduction}

The notion of 1-motive is introduced by Deligne in [3]. A 1-motive over a base scheme $S$ is a two-term complex

$$
M=[Y \stackrel{u}{\rightarrow} G]
$$

of group schemes over $S$ such that

(1) $Y$ sits in degree -1 and is étale locally isomorphic to a finitely generated free abelian group (we call such a group scheme $Y$ a lattice);

(2) $G$ sits in degree 0 and is an extension of an abelian scheme $B$ by a torus $T$ over $S$.

From the definition, one sees that a 1-motive over a field is a mixture of lattice, torus and abelian variety and can be regarded as a mixed motive of weights $0,-1$ and -2 . The name 1-motive comes from the fact that they are the mixed motives arising from varieties of dimension less or equal to 1 . For any positive integer $n$, one can associate to $M$ a finite flat group scheme

$$
T_{\mathbb{Z} / n \mathbb{Z}}(M):=H^{-1}\left(M \otimes^{L} \mathbb{Z} / n \mathbb{Z}\right) .
$$

Now let $R$ be a complete discrete valuation ring with fraction field $K$ and a chosen uniformiser $\pi$, and we endow $S=\operatorname{Spec} R$ with the canonical $\log$ structure; that is, the log structure associated to $\mathbb{N} \rightarrow R, 1 \mapsto \pi$.

According to [13, §4.6.1], a log 1-motive is a triple $M_{\log }=\left(Y, G, u_{K}\right)$, where $Y$ and $G$ are as in the definition of 1-motive and

$$
u_{K}: Y_{K}=Y \times_{S} \operatorname{Spec} K \rightarrow G_{K}=G \times_{S} \operatorname{Spec} K
$$

is a homomorphism of group schemes over $K$. Then we get a 1-motive

$$
M_{K}:=\left[Y_{K} \stackrel{u_{K}}{\longrightarrow} G_{K}\right]
$$

over $K$ out of $M_{\log }$. In [1, Thm. 19], the authors extend $T_{\mathbb{Z} / n \mathbb{Z}}\left(M_{K}\right)$ to a log finite group object in (fin $\left./ S\right)_{r}$ (see Definition 5.1) by using Kato's classification theorem for objects in (fin $/ U)_{\mathrm{r}}$ for an fs $\log$ scheme $U$ with its underlying scheme the spectrum of a noetherian henselian local ring. Note that $Y_{K}$ and $G_{K}$ obviously have good reduction.

In this article, a log 1-motive (see Definition 2.2) is as defined in [8, Def. 2.2] rather than in [13, $\S 4.6 .1]$, which is more suitable over a general base. We are going to show that a 1-motive

$$
M_{K}=\left[Y_{K} \stackrel{u_{K}}{\longrightarrow} G_{K}\right]
$$

with both $Y_{K}$ and $G_{K}$ having good reduction can be extended to a unique log 1-motive

$$
M=\left[Y \stackrel{u}{\rightarrow} G_{\log }\right]
$$

over $S$; see Corollary 3.1. Hence, a $\log 1$-motive in the sense of $[13, \S 4.6 .1]$ is a $\log$ 1-motive in our sense. Taking $T_{\mathbb{Z} / n \mathbb{Z}}(M)$, we get an object of (fin $\left./ S\right)_{\mathrm{r}}$ with generic fibre $T_{\mathbb{Z} / n \mathbb{Z}}\left(M_{K}\right)$. This gives an alternative proof to [1, Thm. 19]. Moreover, we replace log 1-motives by két $\log 1$-motives (see Definition 2.6) and generalise the result to tamely ramified strict 1 -motives over a discrete valuation field $K$ (not necessarily complete); see Theorem 1.1. Here a 1-motive

$$
M_{K}=\left[Y_{K} \stackrel{u_{K}}{\longrightarrow} G_{K}\right]
$$


is called strict if $G_{K}$ has potentially good reduction (see [16, Def. 4.2.3]), and a 1-motive

$$
M_{K}=\left[Y_{K} \stackrel{u_{K}}{\longrightarrow} G_{K}\right]
$$

is called tamely ramified if $Y_{K}$ (respectively $G_{K}$ ) has good reduction (respectively semistable reduction) after a tamely ramified extension of $K$.

Theorem 1.1 (See also Theorem 3.1). Let $K$ be a discrete valuation field with ring of integers $R$, and we endow $S:=\operatorname{Spec} R$ with the canonical log structure. Let

$$
M_{K}=\left[Y_{K} \stackrel{u_{K}}{\longrightarrow} G_{K}\right]
$$

be a tamely ramified strict 1-motive over $K$. Then $M_{K}$ extends to a unique két log 1-motive $M^{\log }$ over $S$. Moreover, the association of $M^{\log }$ to $M_{K}$ gives rise to an equivalence

$$
\text { Két : TameSt-1-Mot } K \text { KétLog-1-Mot }{ }_{S}
$$

from the category of tamely ramified strict 1-motives over $K$ to the category of két log 1-motives over $S$.

Let us make some historical review concerning Theorem 1.1. Without doubt, degeneration is an important topic in mathematics. As stated in the beginning of [8], degenerating abelian varieties cannot preserve smoothness, properness and group structure at the same time, and the theory of log abelian varieties makes the impossible possible ${ }^{1}$ in the world of $\log$ geometry. Let us get back to the setup that $R$ is a complete discrete valuation ring with fraction field $K$ and $S:=\operatorname{Spec} R$ is endowed with the canonical $\log$ structure. Let $A_{K}$ be an abelian variety with semistable reduction over $K$. Following the ideas from [9] and [10], we give a sketch of the construction of the log abelian variety over $S$ extending $A_{K}$. We have the Raynaud extension of $A_{K}$, which is an extension $G$ of some abelian scheme $B$ by some torus $T$ over $S$; see [5, Exp. IX, Prop. 7.5]. Let $A_{K}^{\vee}$ be the dual abelian variety of $A_{K}$. Then $A_{K}^{\vee}$ also has semistable reduction by [5, Exp. IX, Rmk. 3.5.1]. Let $G^{\vee}$ be the Raynaud extension of $A_{K}^{\vee}$, which is an extension of the dual $B^{\vee}$ of $B$ by a torus $T^{\vee}$ by [5, Exp. IX, Prop. 7.5]. Let $Y$ be the character group of $T^{\vee}$. Then the extension $G^{\vee}$ of $B^{\vee}$ by $T^{\vee}$ corresponds to a homomorphism

$$
v: Y \rightarrow\left(B^{\vee}\right)^{\vee}=B .
$$

Let $Y_{K}:=Y \times_{S} \operatorname{Spec} K$ and $G_{K}:=G \times_{S} \operatorname{Spec} K$. Then there exists a homomorphism $u_{K}: Y_{K} \rightarrow G_{K}$ of group schemes over $K$ lifting $v_{K}:=v \times_{S} \operatorname{Spec} K$, such that the rigid analytic space of $A_{K}$ is the quotient of the rigid analytic space of $G_{K}$ by $Y_{K}$; see [5, Exp. IX, §14.1]. So we get a 1-motive

$$
M_{K}=\left[Y_{K} \stackrel{u_{K}}{\longrightarrow} G_{K}\right]
$$

in which both $Y_{K}$ and $G_{K}$ have good reduction (or a log 1-motive in the sense of [13, $\left.\$ 4.6 .1\right]$ ). The 1-motive $M_{K}$ extends to a $\log 1$-motive

$$
M^{\log }=[Y \rightarrow G]
$$

over $S$. Let $\pi$ be a chosen uniformiser of $R, S_{n}:=\operatorname{Spec} R /(\pi)^{n+1}$ endowed with the induced log structure from $S$, and $M_{n}^{\log }:=M^{\log } \times_{S} S_{n}$. Any polarisation $\lambda_{K}$ of $A_{K}$ gives rise to a polarisation $\lambda_{n}$ of $M_{n}^{\log }$. The polarisations $\lambda_{n}$ are compatible with each other as $n$ varies. Let $A_{n}$ be the log abelian variety over

\footnotetext{
${ }^{1}$ To be more precise, one can make the impossible possible up to a certain extent at least at this moment; for example, over a complete discrete valuation field, one can only extend the abelian varieties with semistable reduction to log abelian varieties over the corresponding discrete valuation ring endowed with the canonical log structure.
} 
$S_{n}$ associated to the polarised $\log 1$-motive $M_{n}^{\log }$. Then we get a polarisable $\pi$-adic formal $\log$ abelian variety $\mathcal{A}$ over $R$; that is, an object of the category $\mathcal{P}$ from $[10, \S 5.1]$. Through the equivalence of categories from [10, Thm. 6.1], we get a $\log$ abelian variety $A$ over $S$ whose formal completion is $\mathcal{A}$. To sum up, we have the following associations:

$$
A_{K} \mapsto M_{K} \mapsto M^{\log } \mapsto\left\{M_{n}^{\log }\right\}_{n} \mapsto \mathcal{A} \mapsto A .
$$

Our Theorem 1.1 is a generalisation of the association $M_{K} \mapsto M^{\log }$ to tamely ramified strict 1motives over a discrete valuation field $K$ (not necessarily complete).

In the association chain (1.1), instead of starting with a semi-stable abelian variety over $K$, we can start with a tamely ramified abelian variety over $K$. Then using Theorem 1.1, we can give a proof to the following theorem (see also Theorem 5.2), which is stated in the preprint $[12, \S 4.3]$ without proof.

Theorem 1.2 (Kato). Let $K$ be a complete discrete valuation field with ring of integers $R$, $p$ a prime number and $A_{K}$ a tamely ramified abelian variety over $K$. We endow $S:=\operatorname{Spec} R$ with the canonical log structure. Then the $p$-divisible group $A_{K}\left[p^{\infty}\right]$ of $A_{K}$ extends to a két log $p$-divisible group; that is, an object of $(p-d i v / S)_{\dot{e}}^{\log }$ (see Definition 5.2). It extends to an object of $(p \text {-div } / S)_{\mathrm{d}}^{\log }$ (see Definition 5.2) if any of the following two conditions is satisfied:

(1) $A_{K}$ has semistable reduction.

(2) $p$ is invertible in $R$.

In the association chain (1.1), starting with a tamely ramified abelian variety also brings us the question of whether one can formulate the theory of log abelian varieties in the Kummer étale topology instead of the classical étale topology, with which one could get a complete association chain as in (1.1) in the new case. A second natural question is whether one can go further to define log abelian varieties in the even finer topology the Kummer flat topology. ${ }^{2}$ A third question, as suggested by the anonymous referee, is whether one can extend a $\log 1$-motive $M_{K}$ over an fs $\log$ point with underlying scheme Spec $K$ to a $\log 1$-motive over $\operatorname{Spec} R$ endowed with the direct image log structure along $\operatorname{Spec} K \rightarrow \operatorname{Spec} R$. We hope to come back to these questions in the future.

In Section 2, we define két tori, két lattices, két abelian schemes, két 1-motives and két log 1-motives and formulate duality theory for these objects.

Section 3 is devoted to the proof of Theorem 1.1. A special case of Theorem 1.1 is the following theorem, which gives rise to a concrete nontrivial example of két abelian scheme.

Theorem 1.3 (See also Theorem 3.2). Let $K$ be a discrete valuation field with ring of integers $R$ and $B_{K}$ a tamely ramified abelian variety over $K$ that has potentially good reduction. We endow $S:=\operatorname{Spec} R$ with the canonical log structure. Then $B_{K}$ extends to a unique két abelian scheme $B$ over $S$.

In Section 4 , to a tamely ramified strict 1 -motive $M_{K}$ as in Theorem 1.1 , we associate a logarithmic monodromy pairing and compare it with Raynaud's geometric monodromy for $M_{K}$ (see $[16, \S 4.3])$.

In Section 5, we present a proof to Theorem 1.2.

\section{Két log 1-motives}

\subsection{Két log 1-motives}

The following definitions about log 1-motives are taken from [8, §2].

${ }^{2}$ The theory of $\log$ abelian varieties in the Kummer flat topology has been expected by Kazuya Kato as Professor Chikara Nakayama informed the author. 
Definition 2.1. Let $S$ be an fs $\log$ scheme, $T$ a torus over the underlying scheme of $S$ with character group $X$. The $\log$ augmentation of $T$, denoted by $T_{\log }$, is the sheaf of abelian groups

$$
\mathcal{H}^{\circ} m_{S_{\mathrm{et}}}\left(X, \mathbb{G}_{\mathrm{m}, \log }\right)
$$

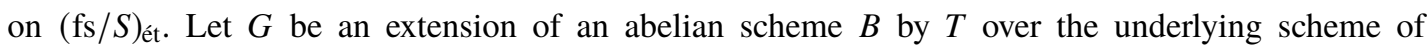
$S$. The logarithmic augmentation of $G$, denoted by $G_{\log }$, is the pushout of $G$ along the inclusion $T \hookrightarrow T_{\log }$.

Definition 2.2. A $\log 1$-motive over an fs $\log$ scheme $S$ is a two-term complex $M=\left[Y \stackrel{u}{\rightarrow} G_{\log }\right]$ in the category of sheaves of abelian groups on (fs $/ S)_{\text {ét }}$, with the degree -1 term $Y$ an étale locally constant sheaf of finitely generated free abelian groups and the degree 0 term $G_{\log }$ as above. We also call $Y$ the lattice part of $M$. A morphism of log 1-motives is just a homomorphism of complexes.

By [19, Prop. 2.1], one can replace (fs $/ S)_{\text {ét }}$ by (fs $\left./ S\right)_{\text {két }}$ in the above definitions. In particular, $T_{\log }$ and $G_{\log }$ are sheaves on $(\text { fs } / S)_{\text {két }}$.

Now we define két 1-motives and két log 1-motives, and we work with (fs/S) két.

Definition 2.3. A két (kummer étale) lattice (respectively két torus, respectively két abelian scheme) over an fs $\log$ scheme $S$ is a sheaf $F$ of abelian groups on (fs $/ S)_{\text {két }}$ such that the pull-back of $F$ to $S^{\prime}$ is a lattice (respectively torus, respectively abelian scheme) over $S^{\prime}$ for some Kummer étale cover $S^{\prime}$ of $S$. Here by a lattice we mean a group scheme that is étale locally representable by a finite rank free abelian group.

Definition 2.4. Let $S$ be an fs $\log$ scheme. A két 1-motive over $S$ is a two-term complex $M=[Y \stackrel{u}{\rightarrow} G]$ in the category of sheaves of abelian groups on (fs $/ S)_{\text {két }}$, such that the degree -1 term $Y$ is a két lattice and the degree 0 term $G$ is an extension of a két abelian scheme $B$ by a két torus $T$ on $(\mathrm{fs} / S)_{\text {két }}$ A morphism of két 1-motives is just a homomorphism of complexes.

Lemma 2.1. Let $S$ be an $f$ s log scheme. Then the associations

$$
T \mapsto \mathcal{H}^{\circ} m_{S_{\text {két }}}\left(T, \mathbb{G}_{\mathrm{m}}\right), \quad X \mapsto \mathcal{H}^{\circ} m_{S_{\text {két }}}\left(X, \mathbb{G}_{\mathrm{m}}\right)
$$

define an equivalence between the category of két tori over $S$ and the category of két locally constant sheaves of finitely generated free abelian groups over $S$, and the equivalence restricts to the classical equivalence between the category of classical tori over $S$ and the category of étale locally constant sheaves of finitely generated free abelian groups over $S$. We call the két lattice $\mathcal{H}{ }_{m_{S_{\mathrm{ket}}}}\left(T, \mathbb{G}_{\mathrm{m}}\right)$ the character group of the két torus $T$.

Proof. This follows from the classical equivalence between the category of tori and the category of étale locally constant sheaves of finitely generated free abelian groups.

Definition 2.5. Given a két torus $T$ over $S$, let $X:=\mathcal{H} o m_{S_{\text {két }}}\left(T, \mathbb{G}_{\mathrm{m}}\right)$ be the character group of $T$. The logarithmic augmentation of $T$, denoted by $T_{\log }$, is the sheaf of abelian groups

$$
\mathcal{H}^{\circ} m_{S_{\text {két }}}\left(X, \mathbb{G}_{\mathrm{m}, \log }\right)
$$

on (fs $/ S)_{\text {két }}$ Let $G$ be an extension of a két abelian scheme $B$ by $T$ over $S$. The logarithmic augmentation of $G$, denoted by $G_{\log }$, is the pushout of $G$ along the inclusion $T \hookrightarrow T_{\log }$.

Note that the quotient $\left(G_{\log } / G\right)_{S_{\text {két }}}$ is canonically identified with the quotient $\left(T_{\log } / T\right)_{S_{\text {két }}}$, which can

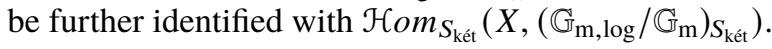

Definition 2.6. A két log 1-motive over an fs $\log$ scheme $S$ is a 2-term complex $M=\left[Y \stackrel{u}{\rightarrow} G_{\log }\right]$ of sheaves of abelian groups on (fs $/ S)_{\text {két }}$ such that the degree -1 term $Y$ is a két lattice over $S$ and $G$ is an 
extension of a két abelian scheme $B$ by a két torus on $(\mathrm{fs} / S)_{\text {két. }}$ The composition

$$
Y \stackrel{u}{\rightarrow} G_{\log } \rightarrow\left(G_{\log } / G\right)_{S_{\text {két }}}=\left(T_{\log } / T\right)_{S_{\text {két }}}=\mathcal{H} \operatorname{om}_{S_{\text {két }}}\left(X,\left(\mathbb{G}_{\mathrm{m}, \log } / \mathbb{G}_{\mathrm{m}}\right)_{S_{\text {két }}}\right)
$$

corresponds to a pairing

$$
Y \times X \rightarrow\left(\mathbb{G}_{\mathrm{m}, \log } / \mathbb{G}_{\mathrm{m}}\right)_{S_{\text {két }}} .
$$

We call this pairing the monodromy pairing of $M$. A morphism of két log 1-motives is just a homomorphism of complexes.

Proposition 2.1. Let $G$ be an extension of a két abelian scheme B by a két torus $T$ over an $f$ s log scheme $S$. Then $G$ is Kummer étale locally an extension of an abelian scheme by a torus for the classical étale topology.

Proof. Without loss of generality, we may assume that $B$ (respectively $T$ ) is an abelian scheme (respectively torus) over $S$. Let $\varepsilon:(\mathrm{fs} / S)_{\text {két }} \rightarrow(\mathrm{fs} / S)_{\text {ét }}$ be the forgetful map between these two sites. The spectral sequence

$$
E_{2}^{i, j}=\operatorname{Ext}_{S_{\mathrm{et}}}^{i}\left(B, R^{j} \varepsilon_{*} T\right) \Rightarrow \operatorname{Ext}_{S_{\mathrm{ket}}}^{i+j}(B, T)
$$

gives rise to an exact sequence

$$
0 \rightarrow \operatorname{Ext}_{S_{\text {ét }}}^{1}(B, T) \rightarrow \operatorname{Ext}_{S_{\text {két }}}^{1}(B, T) \rightarrow \operatorname{Hom}_{S_{\text {ét }}}\left(B, R^{1} \varepsilon_{*} T\right)
$$

By this exact sequence, it suffices to show that $\operatorname{Hom}_{S_{\mathrm{ett}}}\left(B, R^{1} \varepsilon_{*} T\right)=0$. We may assume that $T=\mathbb{G}_{\mathrm{m}}$, then we are reduced to show that $\operatorname{Hom}_{S_{\mathrm{et}}}\left(B, R^{1} \varepsilon_{*} \mathbb{G}_{\mathrm{m}}\right)$ vanishes. The proof of the vanishing is an adoption of the proof of [8, Lem. 6.1.1] in our situation. Letting $\varphi \in \operatorname{Hom}_{S_{\mathrm{et}}}\left(B, R^{1} \varepsilon_{*} \mathbb{G}_{\mathrm{m}}\right)$ and $U$ any object of (fs/S), we show that the map $B(U) \rightarrow R^{1} \varepsilon_{*} \mathbb{G}_{\mathrm{m}}(U)$ induced by $\varphi$ is trivial. By the same argument as in the proof of $[8$, Lem. 6.1.1], we are reduced to the case that $U$ is a $\log$ point; that is, its underlying scheme is the spectrum of a field $K$. Let $p$ be the characteristic of $K$. Then we have

$$
R^{1} \varepsilon_{*} \mathbb{G}_{\mathrm{m}} \cong\left(\mathbb{G}_{\mathrm{m}, \log } / \mathbb{G}_{\mathrm{m}}\right)_{U_{\mathrm{ett}}} \otimes_{\mathbb{Z}}(\mathbb{Q} / \mathbb{Z})^{\prime}
$$

over $U$, where

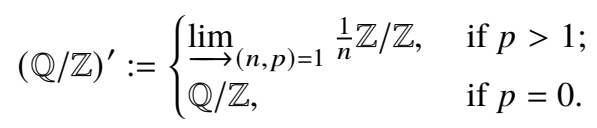

Let $(\mathrm{st} / U)$ be the full subcategory of (fs/U) consisting of all objects that are strict over $U$. The restriction of $\left(\mathbb{G}_{\mathrm{m}, \log } / \mathbb{G}_{\mathrm{m}}\right)_{U_{\mathrm{et}}} \otimes_{\mathbb{Z}}(\mathbb{Q} / \mathbb{Z})^{\prime}$ to $(\mathrm{st} / U)$ is a locally constant sheaf and hence is represented by an étale group scheme over $U$. Because a homomorphism of group schemes over $U$ from $B \times{ }_{S} U$ to an étale group scheme is trivial, the restriction of $\varphi$ to (st/U) is trivial. Hence, the homomorphism $B(U) \rightarrow R^{1} \varepsilon_{*} \mathbb{G}_{\mathrm{m}}(U)$ induced by $\varphi$ is trivial. This finishes the proof.

Remark 2.1. For an abelian scheme $B$ and a torus $T$ over $S$, the same argument as in the proof of Proposition 2.1 shows that $\operatorname{Ext}_{S_{\mathrm{fl}}}^{1}(B, T) \stackrel{\cong}{\rightarrow} \operatorname{Ext}_{S_{\mathrm{kfl}}}^{1}(B, T)$. Furthermore, we have

$$
\operatorname{Ext}_{S_{\text {két }}}^{1}(B, T) \cong \operatorname{Ext}_{S_{\text {et }}}^{1}(B, T) \cong \operatorname{Ext}_{S_{\mathrm{fl}}}^{1}(B, T) \cong \operatorname{Ext}_{S_{\mathrm{kfl}}}^{1}(B, T) \text {. }
$$

\subsection{Két log 1-motives in the Kummer flat topology}

In this subsection, we assume that the underlying scheme of the base $S$ is locally noetherian. We show that a két log 1-motive can be regarded as a 2-term complex in the category of sheaves for the Kummer flat topology. 
Lemma 2.2. Let $S$ be an $f$ s log scheme, and let $F$ be a sheaf of abelian groups on $(\mathrm{fs} / S)_{\text {két }}$ such that $F \times_{S} S^{\prime}$ is representable by an fs log scheme for some Kummer étale cover $S^{\prime}$ of $S$. Then $F$ is also a sheaf for the Kummer flat topology. In particular, két lattices, két tori and két abelian schemes over $S$ are sheaves for the Kummer flat topology.

Proof. It suffices to prove that, for any $U \in(\mathrm{fs} / S)$ and any Kummer flat cover $\left\{U_{i}\right\}_{i \in I}$ of $U$, the canonical sequence

$$
0 \rightarrow F(U) \rightarrow \prod_{i \in I} F\left(U_{i}\right) \rightarrow \prod_{i, j \in I} F\left(U_{i j}\right)
$$

is exact, where $U_{i j}:=U_{i} \times_{U} U_{j}$. Let $S^{\prime \prime}$ denote $S^{\prime} \times_{S} S^{\prime}$. Consider the following commutative diagram:

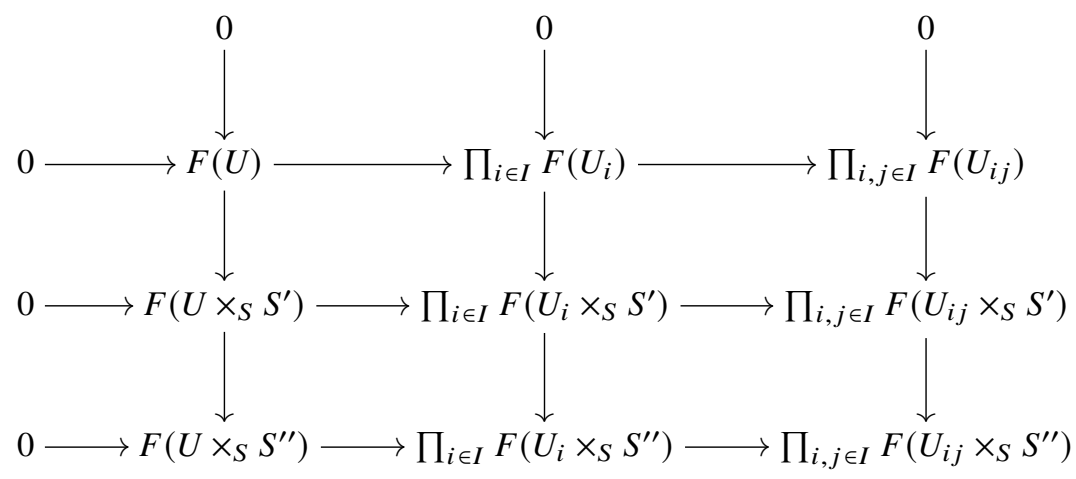

with exact columns. Because $F \times_{S} S^{\prime}$ is representable by an fs $\log$ scheme, so is $F \times_{S} S^{\prime \prime}$. By [9, Thm. 5.2], both $F \times_{S} S^{\prime}$ and $F \times{ }_{S} S^{\prime \prime}$ are sheaves for the Kummer flat topology. It follows that the second row and the third row are both exact. Therefore, the first row is also exact. This finishes the proof.

Corollary 2.1. Let $S$ be an $f$ s log scheme, and let $G$ be an extension of a két abelian scheme B by a két torus $T$ over $S$. Then the logarithmic augmentation $G_{\log }$ of $G$ defined in Definition 2.5 is a sheaffor the Kummer flat topology.

Proof. Because $\mathbb{G}_{\mathrm{m}, \log }$ is a sheaf for the Kummer flat topology by [11, Thm. 3.2] and $X$ is a sheaf for the Kummer flat topology by Lemma 2.2, so is $T_{\log }=\mathcal{H} \operatorname{Hom}_{S_{\text {két }}}\left(X, \mathbb{G}_{\mathrm{m}, \log }\right)$. Let $\delta:(\mathrm{fs} / S)_{\mathrm{kfl}} \rightarrow(\mathrm{fs} / S)_{\text {két }}$ be the forgetful map between these two sites. The adjunction $\left(\delta^{*}, \delta_{*}\right)$ gives rise to the following commutative diagram:

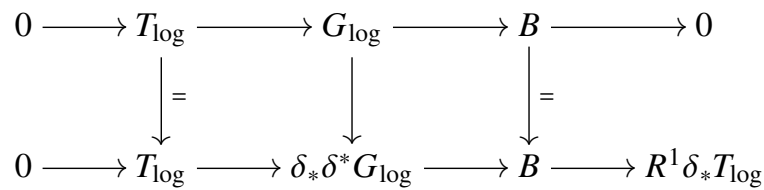

with exact rows. The left vertical identification comes from $T_{\log }$ being a sheaf for the Kummer flat topology. The right vertical identification follows from Lemma 2.2. Because $T_{\log }$ is Kummer étale locally of the form $\mathbb{G}_{\mathrm{m}, \log }^{r}$, we get $R^{1} \delta_{*} T_{\log }=0$ by Kato's logarithmic Hilbert 90 , see [11, §5]. Therefore, the canonical map $G_{\log } \rightarrow \delta_{*} \delta^{*} G_{\log }$ is an isomorphism; that is, $G_{\log }$ is also a sheaf for the Kummer flat topology.

\subsection{Duality of két abelian schemes}

In this subsection we assume that the underlying scheme of the base $S$ is locally noetherian. We formulate the duality theory for két abelian schemes. 
Let $B$ be an abelian scheme over a base scheme $S$. The dual abelian scheme $B^{\vee}$ of $B$ can be described as $\mathcal{E x t}_{S_{\mathrm{fl}}}^{1}\left(B, \mathbb{G}_{\mathrm{m}}\right)$ by the Weil-Barsotti formula. We are going to use this description to define the dual of a given két abelian scheme.

Theorem 2.1. Let $S$ be an $f_{s} \log$ scheme. For any két abelian scheme $B$ over $S$, let $B^{\vee}:=\mathcal{E}_{x} t_{S_{\mathrm{kff}}}^{1}\left(B, \mathbb{G}_{\mathrm{m}}\right)$. Then we have the following:

(1) The sheaf $B^{\vee}$ is a két abelian scheme over $S$.

(2) There exists a functorial isomorphism $\iota: B \stackrel{\cong}{\rightarrow}\left(B^{\vee}\right)^{\vee}$.

Proof. For part (1), we may assume that $B$ is actually an abelian scheme. Let $\varepsilon_{\mathrm{fl}}:(\mathrm{fs} / S)_{\mathrm{kfl}} \rightarrow(\mathrm{fs} / S)_{\mathrm{fl}}$ be the forgetful map between these two sites. Let $F_{1}$ (respectively $F_{2}$ ) be a sheaf on $(\mathrm{fs} / S)_{\mathrm{fl}}$ (respectively $\left.(\mathrm{fs} / S)_{\mathrm{kfl}}\right)$. Then we have

$$
\varepsilon_{\mathrm{fl} *} \mathcal{H}^{\circ} m_{S_{\mathrm{kfl}}}\left(\varepsilon_{\mathrm{fl}}^{*} F_{1}, F_{2}\right)=\mathcal{H} \operatorname{com}_{S_{\mathrm{fl}}}\left(F_{1}, \varepsilon_{\mathrm{ff} *} F_{2}\right) .
$$

Let $\theta$ be the functor sending $F_{2}$ to

$$
\varepsilon_{\mathrm{fl} *} \mathcal{H}_{o} m_{S_{\mathrm{kfl}}}\left(\varepsilon_{\mathrm{fl}}^{*} F_{1}, F_{2}\right)=\mathcal{H} \operatorname{Com}_{S_{\mathrm{fl}}}\left(F_{1}, \varepsilon_{\mathrm{fl} *} F_{2}\right) .
$$

Then we get two Grothendieck spectral sequences

$$
E_{2}^{p, q}=R^{p} \varepsilon_{\mathrm{fl} *} \circ R^{q} \mathcal{H}{ }_{o m} m_{S_{\mathrm{kfl}}}\left(\varepsilon_{\mathrm{fl}}^{*} F_{1},-\right) \Rightarrow R^{p+q} \theta
$$

and

$$
E_{2}^{p, q}=R^{p} \mathcal{H}_{o m_{S_{\mathrm{fl}}}}\left(F_{1},-\right) \circ R^{q} \varepsilon_{\mathrm{fl} *} \Rightarrow R^{p+q} \theta
$$

These two spectral sequences give rise to two exact sequences

$$
\begin{aligned}
0 & \rightarrow R^{1} \varepsilon_{\mathrm{fl} *} \mathcal{H}_{o m_{S_{\mathrm{kfl}}}}\left(\varepsilon_{\mathrm{fl}}^{*} F_{1}, F_{2}\right) \rightarrow R^{1} \theta\left(F_{2}\right) \rightarrow \varepsilon_{\mathrm{ff} *} \mathcal{E}_{x} t_{S_{\mathrm{kfl}}}^{1}\left(\varepsilon_{\mathrm{ff}}^{*} F_{1}, F_{2}\right) \\
& \rightarrow R^{2} \varepsilon_{\mathrm{fl} *} \mathcal{H}_{\mathcal{O}_{\mathrm{kfl}}}\left(\varepsilon_{\mathrm{fl}}^{*} F_{1}, F_{2}\right)
\end{aligned}
$$

and

$$
0 \rightarrow \mathcal{E x t}_{S_{\mathrm{fl}}}^{1}\left(F_{1}, \varepsilon_{\mathrm{fl} *} F_{2}\right) \rightarrow R^{1} \theta\left(F_{2}\right) \rightarrow \mathcal{H} \mathrm{om}_{S_{\mathrm{fl}}}\left(F_{1}, R^{1} \varepsilon_{\mathrm{fl} *} F_{2}\right)
$$

Let $F_{1}=B$ and $F_{2}=\mathbb{G}_{\mathrm{m}}$. Because $\mathcal{H}_{o m_{\mathrm{kfl}_{\mathrm{f}}}}\left(B, \mathbb{G}_{\mathrm{m}}\right)=0$ by [5, Exp. VIII, §3.2.1], we get

$$
R^{1} \theta\left(\mathbb{G}_{\mathrm{m}}\right) \cong \varepsilon_{\mathrm{fl} *} \mathcal{E} x t_{S_{\mathrm{kfl}}}^{1}\left(B, \mathbb{G}_{\mathrm{m}}\right)
$$

therefore, we get an exact sequence

$$
0 \rightarrow \mathcal{E} x t_{S_{\mathrm{fl}}}^{1}\left(B, \mathbb{G}_{\mathrm{m}}\right) \rightarrow \varepsilon_{\mathrm{ff} *} \mathcal{E} x t_{S_{\mathrm{kfl}}}^{1}\left(B, \mathbb{G}_{\mathrm{m}}\right) \rightarrow \mathcal{H} \mathcal{H} m_{S_{\mathrm{fl}}}\left(B, R^{1} \varepsilon_{\mathrm{ff} * \mathbb{G}_{\mathrm{m}}}\right)
$$

We also have

$$
\mathcal{H} \operatorname{om}_{S_{\mathrm{fl}}}\left(B, R^{1} \varepsilon_{\mathrm{fl} *} \mathbb{G}_{\mathrm{m}}\right)=\mathcal{H} \operatorname{Com}_{S_{\mathrm{fl}}}\left(B,\left(\mathbb{G}_{\mathrm{m}, \log } / \mathbb{G}_{\mathrm{m}}\right)_{S_{\mathrm{fl}}} \otimes_{\mathbb{Z}}(\mathbb{Q} / \mathbb{Z})\right)=0
$$

by a similar argument as in the proof of Proposition 2.1. It follows that

$$
\mathcal{E} x t_{S_{\mathrm{fl}}}^{1}\left(B, \mathbb{G}_{\mathrm{m}}\right) \stackrel{\cong}{\rightrightarrows} \varepsilon_{\mathrm{fl} *} \mathcal{E} x t_{S_{\mathrm{kfl}}}^{1}\left(B, \mathbb{G}_{\mathrm{m}}\right) .
$$


By the Weil-Barsotti formula, the sheaf $\mathcal{E} x t_{S_{\mathrm{fl}}}^{1}\left(B, \mathbb{G}_{\mathrm{m}}\right)$ is representable by the dual abelian scheme of $B$. This finishes the proof of part (1).

Now we prove part (2). By [5, Exp. VIII, §3.2.1], we have

$$
\mathcal{H} \operatorname{mom}_{S_{\mathrm{kfl}}}\left(B, \mathbb{G}_{\mathrm{m}}\right)=\mathcal{H} \mathrm{om}_{S_{\mathrm{kfl}}}\left(B^{\vee}, \mathbb{G}_{\mathrm{m}}\right)=0 .
$$

By [5, Exp. VIII, §1.1.1, §1.1.4], we get

$$
\operatorname{Hom}_{S_{\mathrm{kfl}}}\left(B,\left(B^{\vee}\right)^{\vee}\right) \stackrel{\cong}{\leftrightarrows} \operatorname{Biext}_{S_{\mathrm{kfl}}}^{1}\left(B, B^{\vee} ; \mathbb{G}_{\mathrm{m}}\right) \stackrel{\cong}{\rightarrow} \operatorname{Hom}_{S_{\mathrm{kfl}}}\left(B^{\vee}, B^{\vee}\right)
$$

Let $\iota: B \rightarrow\left(B^{\vee}\right)^{\vee}$ be the homomorphism corresponding to $1_{B^{\vee}}$ under the above identification. Note that $\iota$ is the isomorphism giving the duality in the case that $B$ is actually an abelian scheme. Because $B$ is Kummer étale locally an abelian scheme, $\iota$ is Kummer étale locally an isomorphism. It follows that $\iota$ is also an isomorphism over $S$.

Definition 2.7. Let $S$ be an fs $\log$ scheme and $B$ a két abelian scheme over $S$. In view of Theorem 2.1, we call $B^{\vee}:=\mathcal{E} x t_{S_{\mathrm{kfl}}}^{1}\left(B, \mathbb{G}_{\mathrm{m}}\right)$ the dual két abelian scheme of $B$. The biextension $P \in \operatorname{Biext}_{S_{\mathrm{kfl}}}^{1}\left(B, B^{\vee} ; \mathbb{G}_{\mathrm{m}}\right)$ corresponding to $\iota$ is called the Poincaré biextension of $\left(B, B^{\vee}\right)$ by $\mathbb{G}_{\mathrm{m}}$.

Remark 2.2. In view of (2.1), one can also define the dual of $B$ in the flat topology.

\subsection{Duality of két 1-motives}

In this subsection we keep assuming that the underlying scheme of the base $S$ is locally noetherian. We formulate the duality theory for két 1-motives.

First we give an equivalent description of két 1-motives using Poincaré biextension, through which we present the duality theory of két 1-motives. We will also describe morphisms of két 1-motives with respect to the new description. The situation is almost the same as in the case of classical 1-motives, see $[3, \S 10.2 .12, \S 10.2 .13, \S 10.2 .14]$.

Let $M=[Y \stackrel{u}{\rightarrow} G]$ be a két 1-motive over $S$, where $G$ is an extension $0 \rightarrow T \rightarrow G \rightarrow B \rightarrow 0$ of a két abelian scheme $B$ by a két torus $T$ on (fs $/ S)_{\mathrm{kfl}}$. For any element $\chi \in X:=\mathcal{H} o_{S_{\mathrm{kfl}}}\left(T, \mathbb{G}_{\mathrm{m}}\right)$, the pushout of the short exact sequence $0 \rightarrow T \rightarrow G \rightarrow B \rightarrow 0$ along $\chi$ gives rise to an element of $B^{\vee}=\mathcal{E}_{x t_{\mathrm{kff}}}^{1}\left(B, \mathbb{G}_{\mathrm{m}}\right)$, whence a homomorphism

$$
v^{\vee}: X \rightarrow B^{\vee}
$$

Let $v$ be the composition

$$
v: Y \stackrel{u}{\rightarrow} G \rightarrow B
$$

then $u$ corresponds to a unique section $s: Y \rightarrow v^{*} G$ of the extension $v^{*} G \in \operatorname{Ext}_{S_{\mathrm{kfl}}}^{1}(Y, T)$. Consider the following commutative diagram:

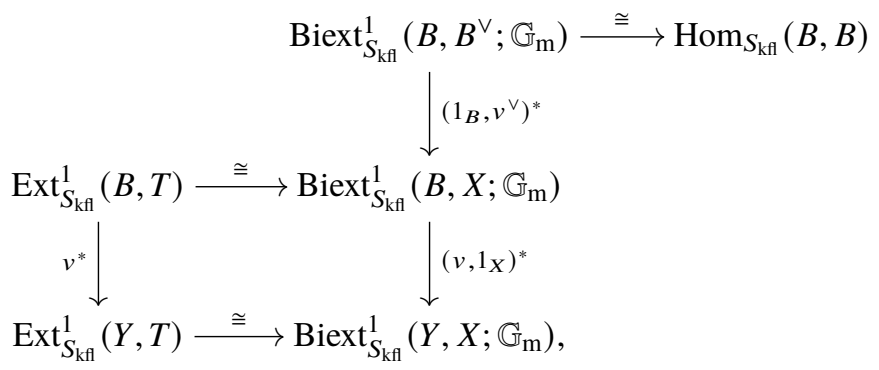


where the horizontal isomorphisms come from

$$
\mathcal{H}^{\circ o m_{S_{\mathrm{kfl}}}}\left(B, \mathbb{G}_{\mathrm{m}}\right)=0=\mathcal{E} x t_{S_{\mathrm{kfl}}}^{1}\left(X, \mathbb{G}_{\mathrm{m}}\right)
$$

and $\mathcal{E} x t_{S_{\mathrm{kff}}}^{1}\left(B^{\vee}, \mathbb{G}_{\mathrm{m}}\right)=B$ with the help of [5, Exp. VIII, §1.1.4]. Because $G$ gives rise to the map $v^{\vee}$, the biextension corresponding to $G$ is $\left(1_{B}, v^{\vee}\right)^{*} P$, and we have the following mapping diagram:

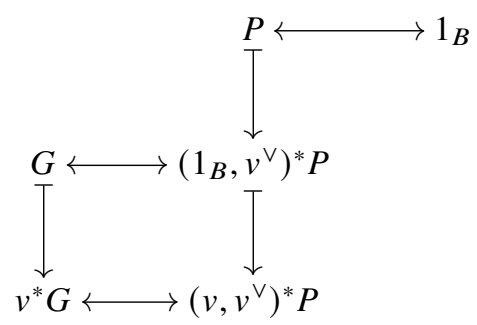

with respect to the commutative diagram (2.4). The section $s$ of $v^{*} G$ corresponds to a section of the biextension $\left(v, v^{\vee}\right)^{*} P$ of $(Y, X)$ by $\mathbb{G}_{\mathrm{m}}$, which we still indicate by $s$ by abuse of notation. Therefore, we get an equivalent description of the két 1-motive $M=[Y \stackrel{u}{\rightarrow} G]$ of the form

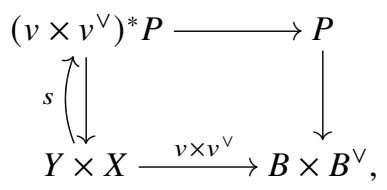

where $\left(v \times v^{\vee}\right)^{*} P$ denotes the pull-back of the Poincare biextension $P$. Note that the section $s$ and the composition

$$
Y \times X \rightarrow\left(v \times v^{\vee}\right)^{*} P \rightarrow P
$$

determine each other; thus, we also denote the composition by $s$ by abuse of notation and use the diagram

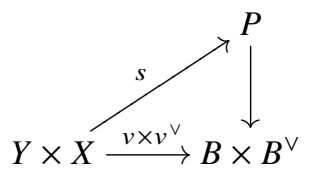

to describe $M$. The description (2.5) is symmetric. If we switch the role of $Y$ and $X, v$ and $v^{\vee}, B$ and $B^{\vee}$, we get another két 1-motive $M^{\vee}=\left[X \stackrel{u^{\vee}}{\longrightarrow} G^{\vee}\right]$, where

$$
G^{\vee} \in \operatorname{Ext}_{S_{\mathrm{kfl}}}^{1}\left(B^{\vee}, T^{\vee}\right)
$$

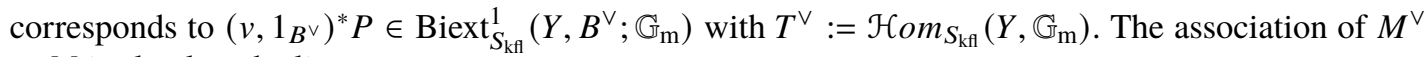
to $M$ is clearly a duality.

Definition 2.8. We call the két 1-motive $M^{\vee}=\left[X \stackrel{u^{\vee}}{\longrightarrow} G^{\vee}\right]$ the dual két 1-motive of the két 1-motive $M=[Y \stackrel{u}{\rightarrow} G]$.

We give a description of morphisms of két 1-motives via Poincaré biextension. Let

$$
\left(f_{-1}, f_{0}\right): M=[Y \stackrel{u}{\rightarrow} G] \rightarrow\left[Y^{\prime} \stackrel{u^{\prime}}{\rightarrow} G^{\prime}\right]=M^{\prime}
$$

be a morphism of két 1-motives. 
Lemma 2.3. There is no nontrivial homomorphism between a két torus and a két abelian scheme.

Proof. This follows from the classical result that there is no nontrivial homomorphism between a torus and an abelian scheme.

By Lemma 2.3, $f_{0}$ induces a map $f_{\mathrm{t}}: T \rightarrow T^{\prime}$ on the torus part and a map $f_{\mathrm{ab}}: B \rightarrow B^{\prime}$ on the abelian part. Let $f_{-1}^{\vee}: X^{\prime} \rightarrow X$ be the map between the character groups induced by $f_{\mathrm{t}}$, and let $f_{\mathrm{ab}}^{\vee}: B^{\prime \vee} \rightarrow B^{\vee}$ be the dual of $f_{\mathrm{ab}}$. Let $v$ (respectively $v^{\vee}$ ) be as in (2.3) (respectively (2.2)), and similarly we define $v^{\prime}$ and $v^{\prime} \vee$ for $M^{\prime}$. Then we get two commutative squares
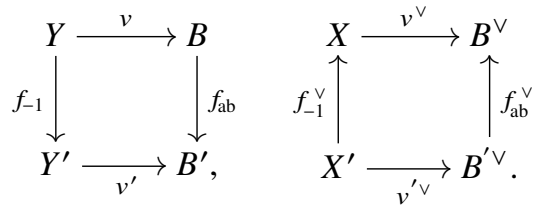

Let $P$ (respectively $P^{\prime}$ ) be the Poincaré biextension of $\left(B, B^{\vee}\right)$ (respectively $\left(B^{\prime}, B^{\prime} \vee\right)$ ) by $\mathbb{G}_{\mathrm{m}}$ and $s: Y \times X \rightarrow P$ (respectively $s^{\prime}: Y^{\prime} \times X^{\prime} \rightarrow P^{\prime}$ ) the section corresponding to $M$ (respectively $M^{\prime}$ ). Then in the following canonical diagram

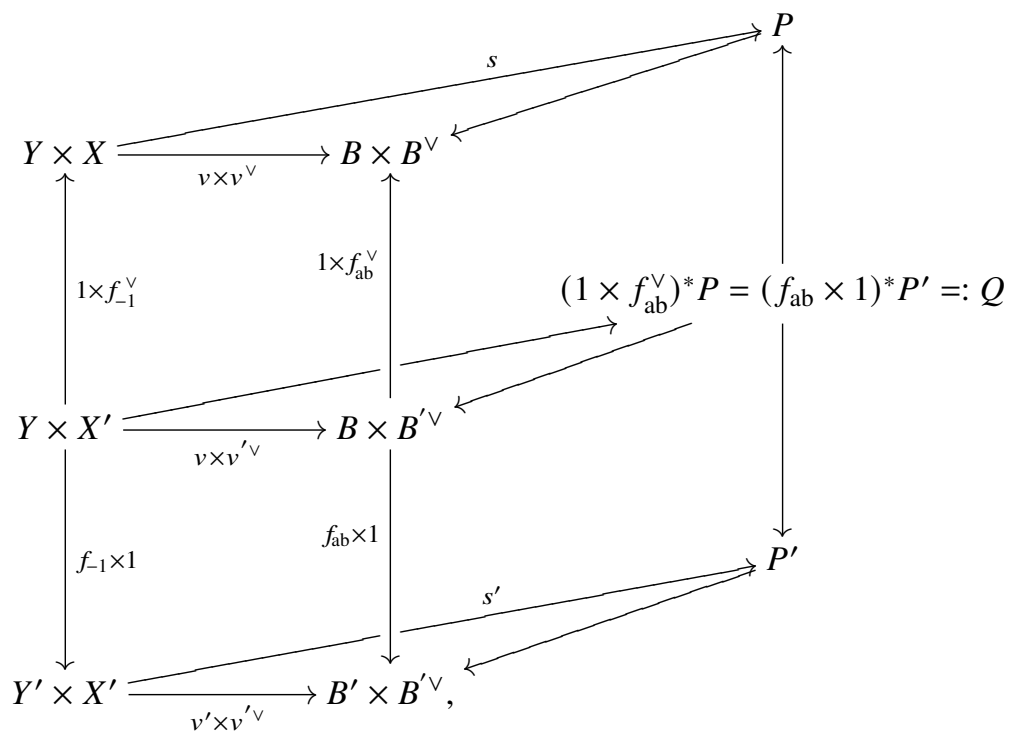

the equality $f_{0} \circ u=u^{\prime} \circ f_{-1}$ implies that for any $y \in Y$ and any $x^{\prime} \in X^{\prime}$ we have

$$
s\left(y, f_{-1}^{\vee}\left(x^{\prime}\right)\right)=s^{\prime}\left(f_{-1}(y), x^{\prime}\right)
$$

after identifying the $\mathbb{G}_{\mathrm{m}}$-torsors $P_{v(y), v^{\vee}\left(f_{-1}^{\vee}\left(x^{\prime}\right)\right)}$ and $P_{v^{\prime}\left(f_{-1}(y)\right), v^{\prime}\left(x^{\prime}\right)}^{\prime}$ along the composition

$$
P_{v(y), v^{\vee}\left(f_{-1}^{\vee}\left(x^{\prime}\right)\right)} \stackrel{\cong}{\longleftarrow} Q_{v(y), v^{\prime} \vee\left(x^{\prime}\right)} \stackrel{\cong}{\stackrel{\longrightarrow}{\longrightarrow}} P_{v^{\prime}\left(f_{-1}(y)\right), v^{\prime} \vee\left(x^{\prime}\right)}
$$

Conversely, given any two commutative squares as in (2.8) such that the equality (2.10) holds with respect to the diagram (2.9), we get a morphism from $M$ to $M^{\prime}$ of két 1-motives.

\subsection{Duality of két log 1-motives}

In this subsection we keep assuming that the underlying scheme of the base $S$ is locally noetherian. We formulate the duality theory for két log 1-motives, which is analogous to the case of két 1-motives. 
First we give an equivalent description of két log 1-motives using Poincaré biextension, through which we present the dual theory of két log 1-motives. We will also describe morphisms of két log 1-motives with respect to the new description.

Let $M=\left[Y \stackrel{u}{\rightarrow} G_{\log }\right]$ be a két $\log 1$-motive over $S$, where $G$ is an extension

$$
0 \rightarrow T \rightarrow G \rightarrow B \rightarrow 0
$$

of a két abelian scheme $B$ by a két torus $T$ on $(\mathrm{fs} / S)_{\mathrm{kfl}}$. For any element $\chi \in X:=\mathcal{H} \operatorname{om}_{S_{\mathrm{kfl}}}\left(T, \mathbb{G}_{\mathrm{m}}\right)$, the pushout of the short exact sequence

$$
0 \rightarrow T \rightarrow G \rightarrow B \rightarrow 0
$$

along $\chi$ gives rise to an element of $B^{\vee}=\mathcal{E} x t_{S_{\mathrm{kfl}}}^{1}\left(B, \mathbb{G}_{\mathrm{m}}\right)$, whence a homomorphism

$$
v^{\vee}: X \rightarrow B^{\vee}
$$

Let $v$ be the composition

$$
v: Y \stackrel{u}{\rightarrow} G_{\log } \rightarrow B
$$

Then $u$ corresponds to a unique section $s: Y \rightarrow v^{*} G_{\log }$ of the extension $v^{*} G_{\log } \in \operatorname{Ext}_{S_{\mathrm{kfl}}}^{1}\left(Y, T_{\log }\right)$. Consider the following commutative diagram:

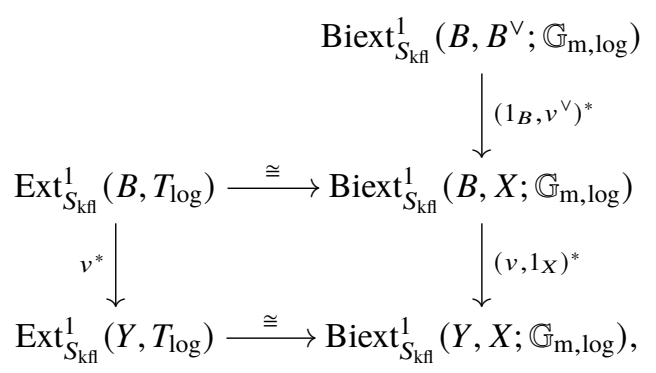

where the horizontal isomorphisms come from

$$
\mathcal{E x t}_{S_{\mathrm{kfl}}}^{1}\left(X, \mathbb{G}_{\mathrm{m}, \log }\right)=0
$$

with the help of [5, Exp. VIII, §1.1.4]. There is an obvious map from the diagram (2.4) to the diagram (2.13). Let $P^{\log }$ be the pushout of $P$ along $\mathbb{G}_{\mathrm{m}} \hookrightarrow \mathbb{G}_{\mathrm{m}}$ log , and we call it the Poincaré biextension of $\left(B, B^{\vee}\right)$ by $\mathbb{G}_{\mathrm{m}, \log }$. Because $G \in \operatorname{Ext}_{S_{\mathrm{kff}}}^{1}(B, T)$ corresponds to the biextension

$$
\left(1_{B}, v^{\vee}\right)^{*} P \in \operatorname{Biext}_{S_{\mathrm{kfl}}}^{1}\left(B, X ; \mathbb{G}_{\mathrm{m}}\right),
$$

we have that $G_{\log } \in \operatorname{Ext}_{S_{\mathrm{kff}}}^{1}\left(B, T_{\log }\right)$ corresponds to

$$
\left(1_{B}, v^{\vee}\right)^{*} P^{\log } \in \operatorname{Biext}_{S_{\mathrm{kfl}}}^{1}\left(B, X ; \mathbb{G}_{\mathrm{m}, \log }\right) .
$$


We have the following mapping diagram:

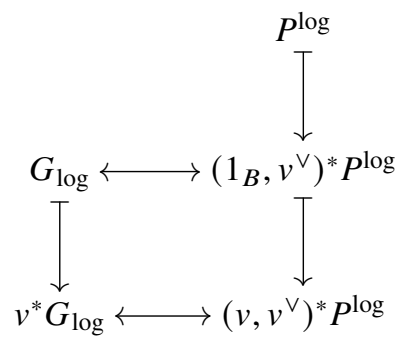

with respect to the commutative diagram (2.13). The section $s$ of $v^{*} G_{\log }$ corresponds to a section of the biextension $\left(v, v^{\vee}\right)^{*} P^{\log }$ of $(Y, X)$ by $\mathbb{G}_{\mathrm{m}}, \log$, which we still denote by $s$ by abuse of notation. Therefore, we get an equivalent description of the két $\log 1$-motive $M=\left[Y \stackrel{u}{\rightarrow} G_{\log }\right]$ of the form

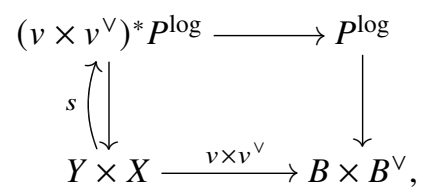

where $\left(v \times v^{\vee}\right)^{*} P^{\log }$ denotes the pull-back of $P^{\log }$ along $v \times v^{\vee}$. Note that the section $s$ and the composition

$$
Y \times X \rightarrow\left(v \times v^{\vee}\right)^{*} P^{\log } \rightarrow P^{\log }
$$

determine each other, and we also denote the composition by $s$ by abuse of notation and use the diagram

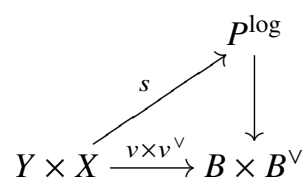

to describe $M$. Note that the description (2.14) is symmetric. If we switch the role of $Y$ and $X, v$ and $v^{\vee}, B$ and $B^{\vee}$, we get another két $\log 1$-motive $M^{\vee}=\left[X \stackrel{u^{\vee}}{\longrightarrow} G_{\log }^{\vee}\right]$, where $G_{\log }^{\vee}$ is the log augmentation of $G^{\vee}$ (see (2.7)). The association of $M^{\vee}$ to $M$ is clearly a duality.

Definition 2.9. We call the két $\log 1$-motive $M^{\vee}=\left[X \stackrel{u^{\vee}}{\rightarrow} G_{\log }^{\vee}\right]$ the dual két log 1-motive of the két $\log$ 1-motive $M=\left[Y \stackrel{u}{\rightarrow} G_{\log }\right]$.

We give a description of morphisms of két log 1-motives via Poincaré biextension. Let

$$
\left(f_{-1}, f_{0}\right): M=\left[Y \stackrel{u}{\rightarrow} G_{\log }\right] \rightarrow\left[Y^{\prime} \stackrel{u^{\prime}}{\rightarrow} G_{\log }^{\prime}\right]=M^{\prime}
$$

be a morphism of két log 1-motives over $S$.

Lemma 2.4. The canonical map

$$
\operatorname{Hom}_{S}\left(G, G^{\prime}\right) \rightarrow \operatorname{Hom}_{S}\left(G_{\log }, G_{\log }^{\prime}\right)
$$

is an isomorphism. 
Proof. Let $\tilde{S}$ be a Kummer étale cover of $S$ such that both $G \times_{S} \tilde{S}$ and $G^{\prime} \times_{S} \tilde{S}$ are extensions of a classical abelian scheme by a classical torus over $\tilde{S}$, and let $\tilde{\tilde{S}}:=\tilde{S} \times{ }_{S} \tilde{S}$. In the following commutative diagram

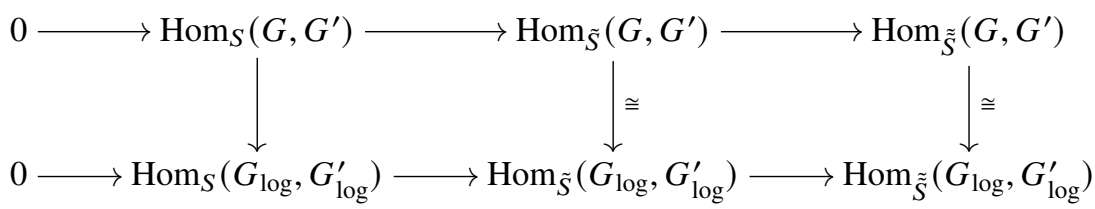

with exact rows, both the middle vertical map and the right vertical map are isomorphisms by [8, Prop. 2.5]. It follows that the left vertical map is an isomorphism.

By Lemma 2.4, $f_{0}$ is induced by a unique homomorphism $f_{\text {sab }}: G \rightarrow G^{\prime}$ over $S$. Let $f_{\mathrm{t}}: T \rightarrow T^{\prime}$ be the map induced by $f_{\text {sab }}$ on the torus part and $f_{-1}^{\vee}: X^{\prime} \rightarrow X$ the map between the character groups induced by $f_{\mathrm{t}}$. Let $f_{\mathrm{ab}}: B \rightarrow B^{\prime}$ be the map induced by $f_{\text {sab }}$ on the abelian part, and let $f_{\mathrm{ab}}^{\vee}: B^{\prime \vee} \rightarrow B^{\vee}$ be the dual of $f_{\mathrm{ab}}$. Let $v$ (respectively $v^{\vee}$ ) be as in (2.12) (respectively (2.11)), and similarly we define $v^{\prime}$ and $v^{\prime} \vee$ for $M^{\prime}$. Then we get two commutative squares

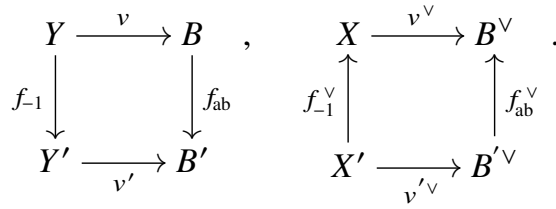

Let $P^{\log }$ (respectively $P^{\prime \log }$ ) be the Poincaré biextension of $\left(B, B^{\vee}\right)$ (respectively $\left(B^{\prime}, B^{\prime} \vee\right)$ ) by $\mathbb{G}_{\mathrm{m}, \log }$ and $s: Y \times X \rightarrow P^{\log }$ (respectively $s^{\prime}: Y^{\prime} \times X^{\prime} \rightarrow P^{\prime} \log$ ) the section corresponding to $M$ (respectively $\left.M^{\prime}\right)$. Then in the following canonical diagram

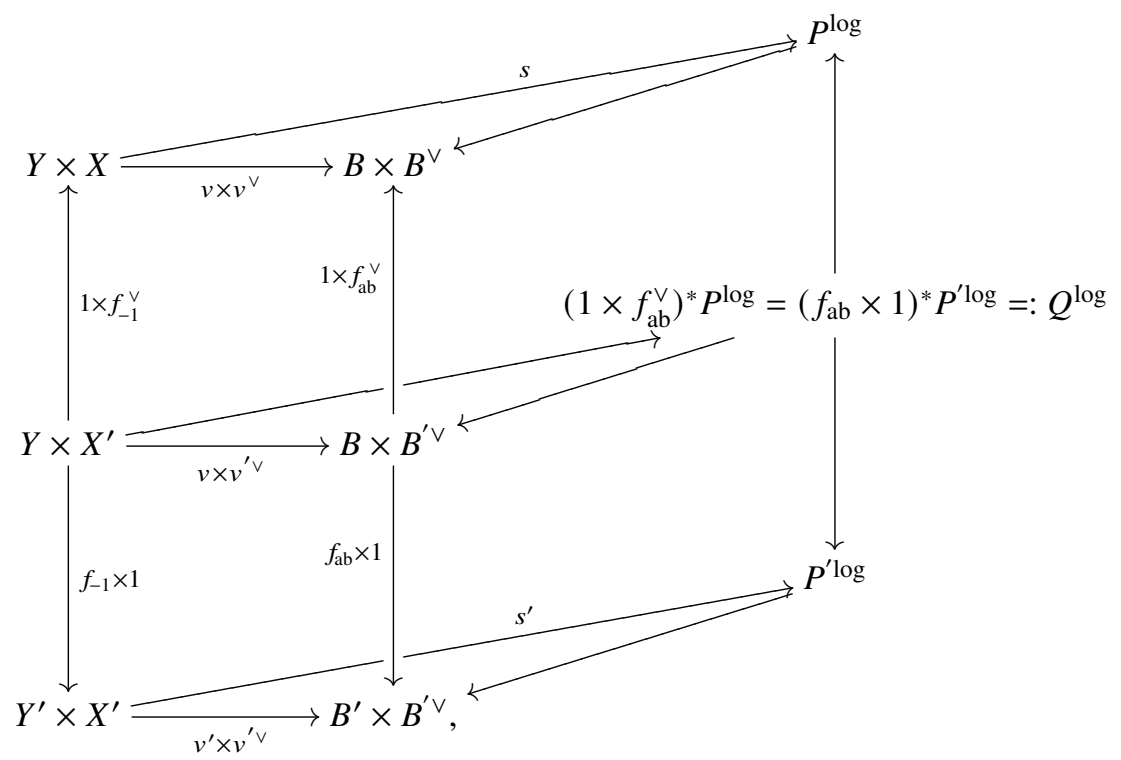

the equality $f_{0} \circ u=u^{\prime} \circ f_{-1}$ implies that for any $y \in Y$ and any $x^{\prime} \in X^{\prime}$ we have

$$
s\left(y, f_{-1}^{\vee}\left(x^{\prime}\right)\right)=s^{\prime}\left(f_{-1}(y), x^{\prime}\right)
$$




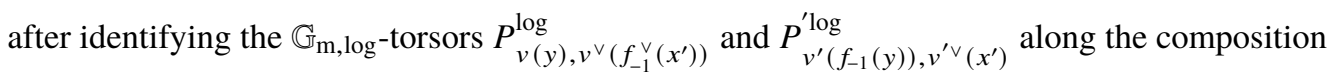

$$
P_{v(y), v^{\vee}\left(f_{-1}^{\vee}\left(x^{\prime}\right)\right)}^{\log } \stackrel{\cong}{\leftrightarrows} Q_{v(y), v^{\prime}\left(x^{\prime}\right)}^{\log } \stackrel{\cong}{\rightarrow} P_{v^{\prime}\left(f_{-1}(y)\right), v^{\prime} \vee\left(x^{\prime}\right)}^{\log }
$$

Conversely, given any two commutative squares as in (2.16) such that the equality (2.18) holds with respect to the diagram (2.17), we get a morphism from $M$ to $M^{\prime}$ of két log 1-motives.

\section{Extending tamely ramified strict 1-motives into két log 1-motives}

From now on, $R$ is a discrete valuation ring with fraction field $K$, residue field $k$ and a chosen uniformiser $\pi, S=\operatorname{Spec} R$, and we endow $S$ with the $\log$ structure associated to $\mathbb{N} \rightarrow R, 1 \mapsto \pi$. Let $s$ (respectively $\eta$ ) be the closed (respectively generic) point of $S$, and we denote by $i: s \hookrightarrow S$ (respectively $j: \eta \hookrightarrow S$ ) the closed (respectively open) immersion of $s$ (respectively $\eta$ ) into $S$. We endow $s$ with the induced $\log$ structure from $S$.

Following [16, Def. 4.2.3], a 1-motive $M_{K}=\left[Y_{K} \stackrel{u_{K}}{\longrightarrow} G_{K}\right]$ over $K$ is called strict if $G_{K}$ has potentially good reduction. We call a 1-motive $M_{K}=\left[Y_{K} \stackrel{u_{K}}{\longrightarrow} G_{K}\right]$ over $K$ tamely ramified if there exists a tamely ramified finite field extension $K^{\prime}$ of $K$ such that $Y_{K} \times_{\operatorname{Spec} K} \operatorname{Spec} K^{\prime}$ has good reduction and $G_{K} \times{ }_{\operatorname{Spec} K}$ Spec $K^{\prime}$ has semistable reduction (i.e., the connected component of the Néron model of $G_{K} \times_{\text {Spec } K}$ Spec $K^{\prime}$ is a semi-abelian scheme). A lattice (respectively torus, respectively abelian variety) over $K$ is called tamely ramified ${ }^{3}$ if it is so regarded as a 1-motive over $K$. A lattice (respectively torus) over $K$ is called unramified if it extends to a lattice (respectively torus) over $S$. The main goal is to prove the following theorem.

Theorem 3.1. Let $K$ be a discrete valuation field with ring of integers $R$, and we endow $S:=\operatorname{Spec} R$ with the canonical log structure. Let $M_{K}=\left[Y_{K} \stackrel{u_{K}}{\longrightarrow} G_{K}\right]$ be a tamely ramified strict 1-motive over $K$ with $G_{K}$ an extension of an abelian variety $B_{K}$ by a torus $T_{K}$. Then $M_{K}$ extends to a unique két log 1-motive $M^{\log }$ over $S$.

Moreover, the association of $M^{\log }$ to $M$ gives rise to an equivalence

$$
\text { Két : TameSt-1-Mot } K \text { KétLog-1-Mot }{ }_{S}
$$

from the category of tamely ramified strict 1-motives over $K$ to the category of két log 1-motives over $S$.

Before going to the proof of Theorem 3.1, we treat some special cases in the first few subsections.

\subsection{Extending tamely ramified lattices into két lattices}

For any positive integer $n$, let $S_{n}$ be the fibre product $S \times_{\operatorname{Spec} \mathbb{Z}[\mathbb{N}]} \operatorname{Spec} \mathbb{Z}[\mathbb{N}]$, where $\operatorname{Spec} \mathbb{Z}[\mathbb{N}]$ is endowed with the $\log$ structure associated to the canonical homomorphism $\mathbb{N} \rightarrow \mathbb{Z}[\mathbb{N}]$ and the map $\operatorname{Spec} \mathbb{Z}[\mathbb{N}] \rightarrow \operatorname{Spec} \mathbb{Z}[\mathbb{N}]$ is induced by the multiplication by $n$ map on the monoid $\mathbb{N}$. The canonical map $S_{n} \rightarrow S$ is a finite Kummer flat cover, and it is even a finite Kummer étale cover if $n$ is invertible on $S$. Let $R_{n}:=R[T] /\left(T^{n}-\pi\right)$. It is easy to see that $R_{n}$ is also a discrete valuation ring and $S_{n}$ is nothing but Spec $R_{n}$ endowed with the $\log$ structure associated to $\mathbb{N} \rightarrow R_{n}, 1 \mapsto T$.

Lemma 3.1. Let $H$ be a két lattice (respectively két torus, respectively két abelian scheme) over $S$. Then

(1) there exists a positive integer $n$ such that $n$ is invertible on $S$ and $H \times_{S} S_{n}$ is a classical lattice (respectively classical torus, respectively classical abelian scheme) over $S_{n}$;

${ }^{3}$ For abelian varieties, this terminology agrees with the one from [6, §2.1.4]. 
(2) the generic fibre $H \times_{S}$ Spec $K$ is a classical lattice (respectively classical torus, respectively classical abelian variety).

Proof. (1) Let $U \rightarrow S$ be a Kummer étale cover such that $H \times_{S} U$ is a classical lattice (respectively classical torus, respectively classical abelian scheme) over $U$. By [15, Prop. 2.15], by passing to a further Kummer étale cover of $S$ over $U$, we may assume that $U \rightarrow S$ factorises as $U \rightarrow S_{n} \rightarrow S$ for some positive integer $n$ with $U \rightarrow S_{n}$ a classical étale cover and $n$ invertible on $S$. It suffices to show that $H \times_{S} S_{n}$ is representable by a classical lattice (respectively classical torus, respectively classical abelian scheme) over $S_{n}$. If $H \times_{S} U$ is a classical torus over $U$ that is affine over $U$, so is $H \times_{S} S_{n}$ by descent with respect to the classical étale cover $U \rightarrow S_{n}$. If $H \times_{S} U$ is a classical lattice over $U$, we are reduced to the torus case by Lemma 2.1. Now we assume that $H \times_{S} U$ is a classical abelian scheme over $U$. Because $U \rightarrow S_{n}$ is a classical étale cover, the morphism $H \times_{S} U \rightarrow H \times_{S} S_{n}$ is representable by surjective classical étale morphisms. Hence, $H \times_{S} S_{n}$ is representable by an algebraic space over $S_{n}$ endowed with the inverse image $\log$ structure from $S_{n}$. By [17, Tag 0422], [17, Tag 041W] and [17, Tag 041V], the morphism $H \times_{S} S_{n} \rightarrow S_{n}$ is proper, flat and of finite presentation. Therefore, $H \times_{S} S_{n} \rightarrow S_{n}$ is an abelian algebraic space; see [4, §1.1] for the definition. Because $S_{n}$ is a trait, $H \times_{S} S_{n} \rightarrow S_{n}$ is actually an abelian scheme by [4, §1.1 (b)]. This finishes the proof of part (1).

(2) Let $H_{K}:=H \times_{S} \operatorname{Spec} K, K_{n}:=R_{n} \otimes_{R} K$ and $H_{K_{n}}:=H \times_{S} S_{n} \times_{S_{n}} \operatorname{Spec} K_{n}$. Then $H_{K_{n}}=$ $H_{K} \times_{\operatorname{Spec} K} \operatorname{Spec} K_{n}$. Because Spec $K_{n}$ is a classical étale cover of Spec $K$ and $H_{K_{n}}$ is a classical lattice (respectively classical torus, respectively classical abelian scheme), so is $H_{K}$ by the same argument as in part (1).

Proposition 3.1. Let $Y_{K}$ be a lattice over $K$; that is, a group scheme over $K$ that is étale locally representable by a finite rank free abelian group. Assume that $Y_{K}$ is tamely ramified, then $Y_{K}$ extends to a unique két lattice $Y$ over $S$.

Furthermore, the association of $Y$ to $Y_{K}$ gives rise to an equivalence of categories

$$
\text { TameLat }_{K} \rightarrow \text { KétLat }_{S}, Y_{K} \mapsto Y
$$

with inverse $Y \mapsto Y \times_{S}$ Spec $K$, where TameLat ${ }_{K}$ denotes the category of tamely ramified lattices over Spec $K$ and KétLat $_{S}$ denotes the category of két lattices over $S$. And the equivalence restricts to an equivalence from the category of unramified lattices over $\operatorname{Spec} K$ to the category of classical lattices over $S$.

Proof. Let $K^{\prime}$ be a tamely ramified finite Galois field extension of $K$ such that $Y_{K} \times_{\operatorname{Spec} K} \operatorname{Spec} K^{\prime}$ is unramified. If necessary, by enlarging $K^{\prime}$ by a further unramified extension, we may assume that $Y_{K} \times_{\text {Spec } K}$ Spec $K^{\prime}$ is constant. Let $R^{\prime}$ be the integral closure of $R$ in $K^{\prime}$ and $\pi^{\prime}$ a uniformiser of $R^{\prime}$. We endow $S^{\prime}:=\operatorname{Spec} R^{\prime}$ with the $\log$ structure associated to $\mathbb{N} \rightarrow R^{\prime}, 1 \mapsto \pi^{\prime}$. Then $S^{\prime}$ is a finite Kummer étale Galois cover of $S$ with Galois group $\operatorname{Gal}\left(K^{\prime} / K\right)$. Therefore, $Y_{K}$ extends to a Kummer étale locally constant sheaf $Y$ on $S$.

By Lemma 3.1 (1), any két lattice becomes a classical lattice after base change to a Kummer étale cover $S_{n} \rightarrow S$ for some positive integer $n$. Therefore, it corresponds to a finite rank free abelian group endowed with a continuous $\pi_{1}^{\log }(S)$-action, where $\pi_{1}^{\log }(S)$ denotes the log étale fundamental group; see $[7, \S 4.6]$. On the other hand, an object of TameLat ${ }_{K}$ corresponds to a finite rank free abelian group endowed with a continuous $G_{K}^{\text {tame }}$-action, where $G_{K}^{\text {tame }}$ denotes the tame quotient of the absolute Galois group of $K$. By [18, Chap. 3, §3.3.1, Example (2)] we have a canonical isomorphism $G_{K}^{\operatorname{tame}} \stackrel{\cong}{\rightarrow} \pi_{1}^{\log }(S)$. It follows that the functor

$$
\text { TameLat }_{K} \rightarrow \text { KétLat }_{S}, Y_{K} \mapsto Y
$$

is an equivalence of categories. Clearly, the equivalence restricts to an equivalence from the category of unramified lattices over Spec $K$ to the category of classical lattices over $S$. 
Example 3.1. Let $Y_{K} \in$ TameLat $_{K}$, which is not unramified. Then it extends to a két lattice over $S$, which is not a classical lattice by Proposition 3.1.

\subsection{Extending tamely ramified tori into két tori}

Proposition 3.2. Let $T_{K}$ be a torus over $K$. Assume that $T_{K}$ is tamely ramified, i.e. there exists a tamely ramified finite field extension $K^{\prime}$ of $K$ such that $T_{K} \times_{K} K^{\prime}$ has good reduction. Then $T_{K}$ extends to a két torus $T$ over $S$.

Furthermore, the association of $T$ to $T_{K}$ gives rise to an equivalence of categories

$$
\text { TameTor }_{K} \rightarrow \text { KétTor }_{S}, T_{K} \mapsto T
$$

with inverse $T \mapsto T \times{ }_{S}$ Spec $K$, where TameTor $_{K}$ denotes the category of tamely ramified tori over $\operatorname{Spec} K$ and $\mathrm{KétTor}_{S}$ denotes the category of két tori over $S$. And the equivalence restricts to an equivalence from the category of unramified tori over $\operatorname{Spec} K$ to the category of classical tori over $S$.

Proof. Because the functor

$$
\operatorname{TameTor~}_{K} \rightarrow \text { TameLat }_{K}, T_{K} \mapsto X_{K}:=\mathcal{H}_{\text {om }}^{(\operatorname{Spec} K)_{\text {êt }}}\left(T_{K}, \mathbb{G}_{\mathrm{m}}\right)
$$

is an equivalence of categories, the result follows from Lemma 2.1 and Proposition 3.1.

Example 3.2. Let $T_{K} \in$ TameTor $_{K}$, which is not unramified. Then it extends to a unique két torus over $S$, which is not a classical torus by Proposition 3.2.

\subsection{Extending tamely ramified abelian varieties with potentially good reduction into két abelian schemes}

Let $B_{K}$ be a tamely ramified abelian variety over $K$, which has potentially good reduction, and let $K^{\prime}$ be a tamely ramified finite Galois field extension of $K$ such that $B_{K^{\prime}}:=B_{K} \times_{\operatorname{Spec} K} \operatorname{Spec} K^{\prime}$ has good reduction. Let $R^{\prime}$ be the integral closure of $R$ in $K^{\prime}$. Then $B_{K^{\prime}}$ extends to an abelian scheme $B^{\prime}$ over $S^{\prime}:=\operatorname{Spec} R^{\prime}$. Let $\pi^{\prime}$ be a uniformiser of $R^{\prime}$, and we endow $S^{\prime}$ with the log structure associated to $\mathbb{N} \rightarrow R^{\prime}, 1 \mapsto \pi^{\prime}$. Then $S^{\prime}$ is a finite Galois Kummer étale cover of $S$ with Galois group $\Gamma:=\operatorname{Gal}\left(K^{\prime} / K\right)$. Let $\rho: \Gamma \times S^{\prime} \rightarrow S^{\prime}$ be the canonical action of $\Gamma$ on $S^{\prime}$. Then the morphism

$$
\left(\rho, \mathrm{pr}_{2}\right): \Gamma \times S^{\prime} \rightarrow S^{\prime} \times_{S} S^{\prime}
$$

is an isomorphism. By $\left[2, \S 1.2\right.$, Prop. 8], $B^{\prime}$ is the Néron model of $B_{K^{\prime}}$. By the universal property of Néron models, the $\Gamma$-action on $B_{K^{\prime}}$ extends to a unique $\Gamma$-action

$$
\tilde{\rho}: \Gamma \times B^{\prime} \rightarrow B^{\prime}
$$

on $B^{\prime}$, which is compatible with the $\Gamma$-action $\rho$ on $S^{\prime}$ and the group structure of $B^{\prime}$. We endow $B^{\prime}$ with the induced $\log$ structure from $S^{\prime}$.

Let $p^{\prime}$ denote the structure morphism $B^{\prime} \rightarrow S^{\prime}, \alpha$ denote the morphism $S^{\prime} \rightarrow S$ and $p:=\alpha \circ p^{\prime}$. For any $U \in(\mathrm{fs} / S)$ and any $(a, b) \in\left(B^{\prime} \times{ }_{S} B^{\prime}\right)(U)$, we have $\alpha\left(p^{\prime}(a)\right)=\alpha\left(p^{\prime}(b)\right)$. Hence, there exists a unique $\gamma \in \Gamma$ such that $p^{\prime}(a)=\rho\left(\gamma, p^{\prime}(b)\right)$. Because

$$
p^{\prime}(\tilde{\rho}(\gamma, b))=\rho\left(\gamma, p^{\prime}(b)\right)=p^{\prime}(a),
$$


we get $(a, \tilde{\rho}(\gamma, b)) \in\left(B^{\prime} \times_{S^{\prime}} B^{\prime}\right)(U)$. We define a morphism

$$
\Phi: B^{\prime} \times_{S} B^{\prime} \rightarrow \Gamma \times\left(B^{\prime} \times_{S^{\prime}} B^{\prime}\right)
$$

by sending $(a, b)$ to $\left(\gamma^{-1},(a, \tilde{\rho}(\gamma, b))\right.$.

Lemma 3.2. The morphism $\Phi$ is an isomorphism with inverse

$$
\Psi: \Gamma \times\left(B^{\prime} \times_{S^{\prime}} B^{\prime}\right) \rightarrow B^{\prime} \times_{S} B^{\prime}, \quad(\gamma,(a, b)) \mapsto(a, \tilde{\rho}(\gamma, b))
$$

for any $U \in(\mathrm{fs} / S)$, any $(a, b) \in\left(B^{\prime} \times_{S^{\prime}} B^{\prime}\right)(U)$ and any $\gamma \in \Gamma$.

Proof. Clearly $\Phi$ and $\Psi$ are inverse to each other.

Lemma 3.3. The canonical morphism

$$
\left(\tilde{\rho}, \mathrm{pr}_{2}\right): \Gamma \times B^{\prime} \rightarrow B^{\prime} \times_{S} B^{\prime}
$$

is a monomorphism of sheaves on $(\mathrm{fs} / S)_{\text {két }}$.

Proof. The composition

$$
\Gamma \times B^{\prime} \stackrel{\left(\tilde{\rho}, \mathrm{pr}_{2}\right)}{\longrightarrow} B^{\prime} \times_{S} B^{\prime} \stackrel{\iota}{\rightarrow} B^{\prime} \times_{S} B^{\prime} \stackrel{\Phi}{\rightarrow} \Gamma \times\left(B^{\prime} \times_{S^{\prime}} B^{\prime}\right)
$$

is identified with the morphism $1_{\Gamma} \times \Delta_{B^{\prime} / S^{\prime}}$, where $\iota$ denotes the morphism switching the two factors and $\Delta_{B^{\prime} / S^{\prime}}$ denotes the diagonal embedding. Therefore, the result follows.

By [17, Tag 0234], the action $\tilde{\rho}$ defines a groupoid scheme over $S$; hence, by [17, Tag 0232] the morphism

$$
\left(\tilde{\rho}, \mathrm{pr}_{2}\right): \Gamma \times B^{\prime} \rightarrow B^{\prime} \times_{S} B^{\prime}
$$

is a pre-equivalence relation. Moreover, $\left(\tilde{\rho}, \mathrm{pr}_{2}\right)$ is an equivalence relation by Lemma 3.3. The morphism

$$
\left(\rho, \mathrm{pr}_{2}\right): \Gamma \times S^{\prime} \rightarrow S^{\prime} \times_{S} S^{\prime}
$$

being an isomorphism is clearly an equivalence relation.

Now we are following [17, Tag 02VE] to construct a két abelian scheme over $S$. We remark that although the setup there does not agree with ours, the proofs there work verbatim in our case.

Following the approach of [17, Tag $02 \mathrm{VG}]$, we take the quotient sheaves for the equivalence relations $\left(\tilde{\rho}, \mathrm{pr}_{2}\right)$ and $\left(\rho, \mathrm{pr}_{2}\right)$ on the site $(\mathrm{fs} / S)_{\text {két }}$. Because $\left(\rho, \mathrm{pr}_{2}\right)$ is an isomorphism, the corresponding quotient sheaf is representable by the terminal object $S$. Let $B=\operatorname{Ke} t\left(B_{K}\right)$ be the quotient sheaf for the equivalence relation $\left(\tilde{\rho}, \mathrm{pr}_{2}\right)$. Because the two equivalence relations are compatible with each other, we get a morphism $B \rightarrow S$. Since the equivalence relation $\left(\tilde{\rho}, \mathrm{pr}_{2}\right)$ is compatible with the group structure of $B^{\prime}$, the quotient sheaf $B^{\prime}$ carries a structure of sheaf of abelian groups. The verbatim translations of the proof of [17, Tag 045Y] and the proof of [17, Tag 07S3] show that

$$
\Gamma \times B^{\prime} \stackrel{\cong}{\rightarrow} B^{\prime} \times_{B} B^{\prime}
$$

and

$$
B^{\prime} \stackrel{\cong}{\rightarrow} B \times_{S} S^{\prime}
$$

respectively; hence, $B$ is a két abelian scheme over $S$.

Let $C_{K}$ be another tamely ramified abelian variety over $K$, which has potentially good reduction, and let $f_{K}: B_{K} \rightarrow C_{K}$ be a homomorphism of group schemes over $K$. By enlarging the field $K^{\prime}$ such that $K^{\prime}$ remains a finite tamely ramified extension of $K$, we may assume that $C_{K^{\prime}}:=C_{K} \times_{\operatorname{Spec} K} \operatorname{Spec} K^{\prime}$ extends 
to an abelian scheme $C^{\prime}$ over $S^{\prime}$. By the same reason as for $B_{K}$, we have a natural action of $\Gamma$ on $C^{\prime}$ that is compatible with the natural action of $\Gamma$ on $S^{\prime}$ and the quotient for the action gives rise to a két abelian scheme over $S$. Let $f_{K^{\prime}}$ be the base change of $f_{K}$ to $K^{\prime}$. Then it extends to a unique homomorphism $f^{\prime}: B^{\prime} \rightarrow C^{\prime}$ of group schemes over $S^{\prime}$ by the universal property of the Néron model. For any $\gamma \in \Gamma$, the compatibility of the canonical actions of $\Gamma$ on $B_{K^{\prime}}$ and $C_{K^{\prime}}$ gives rise to a commutative diagram

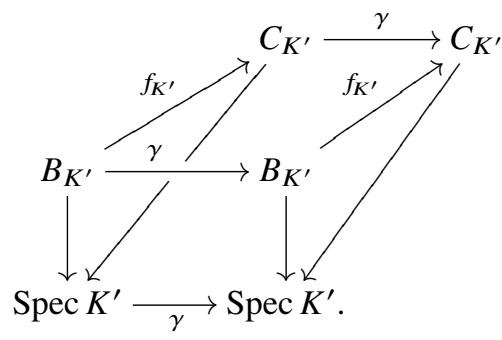

Hence, the following diagram

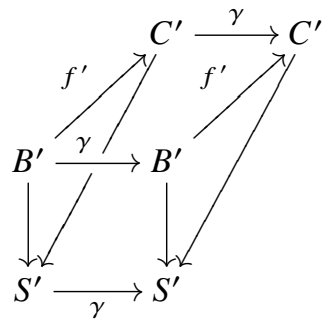

is commutative by the universal property of the Néron model for any $\gamma \in \Gamma$. It follows that the $\Gamma$-actions on $B^{\prime}$ and $C^{\prime}$ are compatible with each other; hence, $f^{\prime}$ induces a homomorphism $f: B \rightarrow C$ of két abelian schemes over $S$, where $C=\operatorname{Két}\left(C_{K}\right)$ is the két abelian scheme for $C_{K}$ defined in the same way as $B=\operatorname{Két}\left(B_{K}\right)$ for $B_{K}$.

Therefore, we get a functor

$$
\text { Két }: \operatorname{TameStAb}_{K} \rightarrow \mathrm{KétAb}_{S}, \quad B_{K} \mapsto \text { Két }\left(B_{K}\right)
$$

from the category of tamely ramified abelian varieties with potentially good reduction over $K$ to the category of két abelian schemes over $S$.

\section{Theorem 3.2.}

(1) Let $B$ and $C$ be two két abelian schemes over $S$, and let $B_{K}:=B \times_{S} \operatorname{Spec} K$ and $C_{K}:=C \times_{S} \operatorname{Spec} K$. Then the restriction map

$$
\operatorname{Hom}_{S}(B, C) \rightarrow \operatorname{Hom}_{\text {Spec } K}\left(B_{K}, C_{K}\right)
$$

is an isomorphism.

(2) The functor

$$
\text { Két }: \text { TameStAb }_{K} \rightarrow \text { KétAb }_{S}, \quad B_{K} \mapsto \text { Két }\left(B_{K}\right)
$$

is an equivalence of categories, and it restricts to an equivalence from the category of abelian varieties with good reduction over $K$ to the category of abelian schemes over $S$.

Proof. (1) By Lemma 3.1, there exists a positive integer $n$ such that both $B_{n}:=B \times{ }_{S} S_{n}$ and $C_{n}:=C \times_{S} S_{n}$ are classical abelian schemes over $S_{n}$, where $S_{n}$ is as defined in the beginning of Subsection 3.1. Let $\tilde{S}_{n}:=S_{n} \times_{S} S_{n}, \tilde{B}_{n}:=B \times_{S} \tilde{S}_{n}, \tilde{C}_{n}:=C \times_{S} \tilde{S}_{n}, K_{n}:=S_{n} \times_{S} \operatorname{Spec} K, \tilde{K}_{n}:=\tilde{S}_{n} \times_{S} \operatorname{Spec} K$, $B_{K_{n}}:=B_{n} \times{ }_{S} \operatorname{Spec} K, C_{K_{n}}:=C_{n} \times{ }_{S} \operatorname{Spec} K, \tilde{B}_{K_{n}}:=\tilde{B}_{n} \times{ }_{S}$ Spec $K$ and $\tilde{C}_{K_{n}}:=\tilde{C}_{n} \times{ }_{S}$ Spec $K$. Because $S_{n}$ is a Kummer étale cover of $S$, we get the following commutative diagram: 


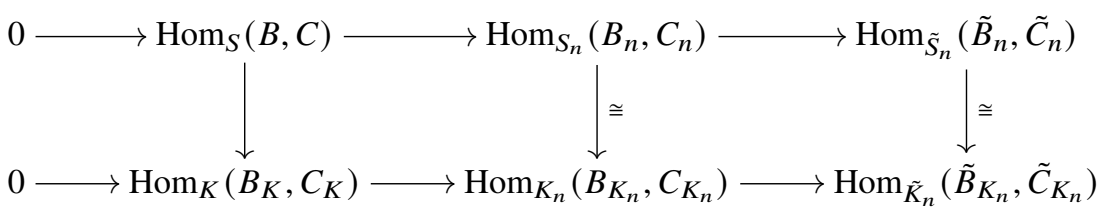

with exact rows. Here we abbreviate $\operatorname{Hom}_{\mathrm{Spec} ?}(-,-)$ as $\operatorname{Hom}_{?}(-,-)$ for $?=K, K_{n}, \tilde{K}_{n}$, in order to make the diagram not too big. The middle vertical map and the right vertical map are isomorphisms by the universal property of Néron models, it follows that the first vertical map is also an isomorphism.

(2) By part (1), the functor Két is fully faithful. Given any $B \in \mathrm{KétAb}_{S}$, we have $B_{K}:=B \times_{S} \operatorname{Spec} K \in$ TameStAb $_{K}$ by Lemma 3.1 (2). By part (1), the identity map on $B_{K}$ extends to a unique isomorphism $K e ́ t\left(B_{K}\right) \stackrel{\cong}{\rightarrow} B$. Hence, the functor $K e ́ t$ is essentially surjective, and so is an equivalence of categories. In fact, it is easy to see that the functor

$$
(-)_{K}: \operatorname{KétAb}_{S} \rightarrow \operatorname{TameStAb}_{K}, B \mapsto B \times_{S} \operatorname{Spec} K
$$

is inverse to the functor Két by the construction of Két. If $A_{K} \in \operatorname{TameStAb}_{K}$ has good reduction - that is, it extends to an abelian scheme $A$ over $S$ (which is unique by the theory of Néron models) - then there exists a unique isomorphism $A \rightarrow \operatorname{Két}\left(A_{K}\right)$ extending $\operatorname{id}_{A_{K}}$. Therefore, Két $\left(A_{K}\right)=A$, and so the functor $K e$ é restricts to an equivalence from the category of abelian varieties with good reduction over $K$ to the category of abelian schemes over $S$.

Example 3.3. Let $B_{K} \in \mathrm{TameStAb}_{K}$, which has no good reduction. Then Két $\left(B_{K}\right)$ is a két abelian scheme over $S$ that is not a classical abelian scheme by Theorem 3.2 (2).

It is natural to investigate whether the functor Két is compatible with the dualities on both sides.

Proposition 3.3. The functor Két : TameStAb $\mathrm{Ta}_{K} \rightarrow \mathrm{KétAb}_{S}$ is compatible with the dualities; that is, we have a canonical identification

$$
\operatorname{Két}\left(B_{K}^{\vee}\right) \cong \operatorname{Két}\left(B_{K}\right)^{\vee} \text {. }
$$

Proof. Let $S^{\prime}, \Gamma, B^{\prime}$ and $B$ be as in (3.3) and (3.4). Then $B=K e ́ t\left(B_{K}\right)$. By (3.4), we have

$$
\begin{aligned}
B^{\vee} \times_{S} S^{\prime} & =\mathcal{E} x t_{S_{\mathrm{kfl}}}^{1}\left(B, \mathbb{G}_{\mathrm{m}}\right) \times_{S} S^{\prime}=\mathcal{E} x t_{S_{\mathrm{kfl}}^{\prime}}^{1}\left(B \times_{S} S^{\prime}, \mathbb{G}_{\mathrm{m}}\right) \\
& =\mathcal{E} x t_{S_{\mathrm{kfl}}^{\prime}}^{1}\left(B^{\prime}, \mathbb{G}_{\mathrm{m}}\right)=B^{\prime \vee} .
\end{aligned}
$$

It follows that $B^{\vee}=\mathcal{E} x t_{S_{\text {két }}}^{1}\left(B, \mathbb{G}_{\mathrm{m}}\right)$ is the quotient sheaf for a descent data with respect to the Galois Kummer étale cover $S^{\prime} / S$. Such a descent data is given by a group action $\tau: \Gamma \times B^{\prime \vee} \rightarrow B^{\prime \vee}$. In order to have the identification $K e ́ t\left(B_{K}^{\vee}\right) \cong K^{2} t\left(B_{K}\right)^{\vee}=B^{\vee}$, we are reduced to identify the action $\tau$ with the action $\tilde{\rho}^{\vee}: \Gamma \times B^{\prime \vee} \rightarrow B^{\prime} \vee$ for $B_{K}^{\vee}$ that corresponds to the action (3.1) for $B_{K}$. But this is clear, because $\Gamma \times B^{\prime \vee}=\sqcup_{\gamma \in \Gamma} B^{\prime \vee}$ and these two actions agree over the generic fibre.

\subsection{Proof of Theorem 3.1}

In this subsection, we prove Theorem 3.1.

Let $v_{K}$ be the composition $Y_{K} \stackrel{u_{K}}{\longrightarrow} G_{K} \rightarrow B_{K}, X_{K}$ the character group of the torus $T_{K}$ and $v_{K}^{\vee}: X_{K} \rightarrow B_{K}^{\vee}$ the homomorphism corresponding to the semi-abelian variety $G_{K}$. By [16, §2.4.1], the 1-motive $M_{K}$ is uniquely determined by a commutative diagram of the form 


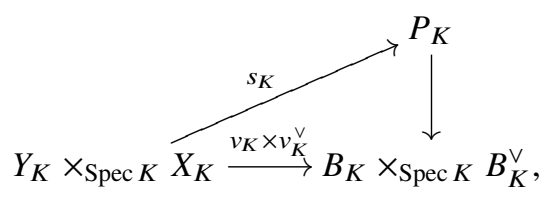

where $s_{K}$ is a bilinear map. Note that $s_{K}$ corresponds to a unique section

$$
t_{K}: Y_{K} \times_{\operatorname{Spec} K} X_{K} \rightarrow E_{K},
$$

where $E_{K}$ denotes the pull-back of the Poincaré biextension $P_{K}$ of $B_{K}$ and its dual $B_{K}^{\vee}$ along $v_{K} \times v_{K}^{\vee}$.

Let $K^{\prime}$ be a finite tamely ramified Galois extension of $K$ such that

$$
B_{K} \times_{\operatorname{Spec} K} \operatorname{Spec} K^{\prime}
$$

extends to an abelian scheme $B^{\prime}$ over $S^{\prime}:=\operatorname{Spec} R^{\prime}$,

$$
Y_{K} \times_{\operatorname{Spec} K} \operatorname{Spec} K^{\prime}
$$

extends to a constant group scheme over $S^{\prime}$ and

$$
T_{K} \times_{\operatorname{Spec} K} \operatorname{Spec} K^{\prime}
$$

extends to a split torus over $S^{\prime}$, where $R^{\prime}$ denotes the integral closure of $R$ in $K^{\prime}$. Let $\pi^{\prime}$ be a uniformiser of $R^{\prime}$ such that $\pi^{\prime}=\pi^{\frac{1}{e}}$ with $e$ the ramification index of the extension $K^{\prime} / K$, and we endow $S^{\prime}$ with the $\log$ structure associated to $\mathbb{N} \rightarrow R^{\prime}, 1 \mapsto \pi^{\prime}$. Then $S^{\prime}$ is a finite Galois Kummer étale cover of $S$ with Galois group $\Gamma:=\operatorname{Gal}\left(K^{\prime} / K\right)$.

Let $Y$ (respectively $X$ ) be the két lattice over $S$ extending $Y_{K}$ (respectively $X_{K}$ ) as constructed in Subsection 3.1. Then $Y$ (respectively $X$ ) can be regarded as a $\Gamma$-module. Let $T$ be the két torus over $S$ extending $T_{K}$ as constructed in Subsection 3.2. Note that $T$ is nothing but $\mathcal{H}_{0} m_{S_{\text {két }}}\left(X, \mathbb{G}_{\mathrm{m}}\right)$. Let $B$ (respectively $B^{\vee}$ ) be the két abelian scheme extending $B_{K}$ (respectively $B_{K}^{\vee}$ ) as constructed in Subsection 3.3, and let $P$ be the Poincaré biextension of $\left(B, B^{\vee}\right)$ by $\mathbb{G}_{\mathrm{m}}$.

Lemma 3.4. The homomorphism $v_{K}$ (respectively $v_{K}^{\vee}$ ) extends to a unique homomorphism $v: Y \rightarrow B$ (respectively $v^{\vee}: X \rightarrow B^{\vee}$ ).

Proof. We only treat the case of $v_{K}$, and the other case can be done in the same way. We have $B \times_{S} S^{\prime}=B^{\prime}$ by (3.4). Therefore,

$$
B\left(S^{\prime}\right)=B^{\prime}\left(S^{\prime}\right)=B^{\prime}\left(\operatorname{Spec} K^{\prime}\right)=B_{K}\left(\operatorname{Spec} K^{\prime}\right) .
$$

Because $Y$ is equivalent to a $\Gamma$-module, we get

$$
\operatorname{Hom}_{S}(Y, B)=\operatorname{Hom}_{\mathbb{Z}-\operatorname{Mod}}\left(Y, B\left(S^{\prime}\right)\right)^{\Gamma}=\operatorname{Hom}_{\mathbb{Z}-\operatorname{Mod}}\left(Y_{K}, B_{K}\left(\operatorname{Spec} K^{\prime}\right)\right)^{\Gamma} .
$$

It follows that $v_{K}$ extends to a unique homomorphism $v: Y \rightarrow B$.

By Lemma 3.4, we get a map $v \times v^{\vee}: Y \times_{S} X \rightarrow B \times_{S} B^{\vee}$. Letting $P^{\log }$ be the pushout of $P$ along the inclusion $\mathbb{G}_{\mathrm{m}} \hookrightarrow \mathbb{G}_{\mathrm{m}, \log }$, we get the following diagram:

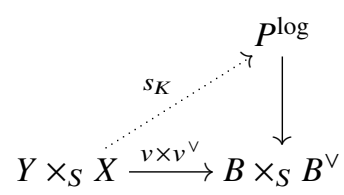

over $S$. The dotted arrow in (3.7) means that it is only a map over $\operatorname{Spec} K$. The restriction of (3.7) to Spec $K$ is clearly just the diagram (3.5). 


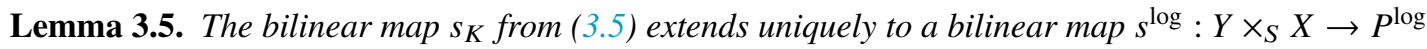
making the diagram (3.7) commutative.

Proof. Let $E$ be the pull-back of $P$ along the map $v \times v^{\vee}$ on (fs $\left./ S\right)_{\mathrm{kfl}}$, and let $E^{\log }$ be the pushout of $E$ along the canonical map $\mathbb{G}_{\mathrm{m}} \hookrightarrow \mathbb{G}_{\mathrm{m}, \log }$ on $(\mathrm{fs} / S)_{\mathrm{kfl}}$. Because both $Y$ and $X$ are Kummer étale locally representable by a finitely generated free abelian group, we have

$$
\operatorname{Biext}_{S_{\mathrm{kfl}}}^{1}(Y, X ;-)=\operatorname{Ext}_{S_{\mathrm{kfl}}}^{1}\left(Y \otimes_{\mathbb{Z}}^{\mathbb{L}} X,-\right)=\operatorname{Ext}_{S_{\mathrm{kfl}}}^{1}\left(Y \otimes_{\mathbb{Z}} X,-\right)
$$

by [5, Exp. VII, §3.6.5]. Therefore, $E$ (respectively $\left.E^{\mathrm{log}}\right)$ can be regarded as an element of $\operatorname{Ext}_{S_{\mathrm{kfl}}}^{1}\left(Y \otimes_{\mathbb{Z}}\right.$ $\left.X, \mathbb{G}_{\mathrm{m}}\right)\left(\right.$ respectively $\left.\operatorname{Ext}_{S_{\mathrm{kfl}}}^{1}\left(Y \otimes_{\mathbb{Z}} X, \mathbb{G}_{\mathrm{m}, \log }\right)\right)$, and $E^{\log }$ is still the pushout of $E$ under these identifications. Similarly, $E_{K}:=E \times_{S} \operatorname{Spec} K$ can be regarded as an element of $\operatorname{Ext}_{(\operatorname{Spec} K)_{\mathrm{fl}}}^{1}\left(Y_{K} \otimes_{\mathbb{Z}} X_{K}, \mathbb{G}_{\mathrm{m}}\right)$. Note that both $E$ and $E^{\log }$ over $S$ restrict to $E_{K}$ over $K$. The extensions $E, E^{\log }$ and $E_{K}$ give rise to exact sequences

$$
\begin{gathered}
0 \rightarrow \mathbb{G}_{\mathrm{m}}\left(S^{\prime}\right) \rightarrow E\left(S^{\prime}\right) \rightarrow Y \otimes_{\mathbb{Z}} X\left(S^{\prime}\right) \rightarrow H_{\mathrm{kfl}}^{1}\left(S^{\prime}, \mathbb{G}_{\mathrm{m}}\right), \\
0 \rightarrow \mathbb{G}_{\mathrm{m}, \log }\left(S^{\prime}\right) \rightarrow E^{\log }\left(S^{\prime}\right) \rightarrow Y \otimes_{\mathbb{Z}} X\left(S^{\prime}\right) \rightarrow H_{\mathrm{kfl}}^{1}\left(S^{\prime}, \mathbb{G}_{\mathrm{m}, \log }\right)
\end{gathered}
$$

and

$$
0 \rightarrow \mathbb{G}_{\mathrm{m}}\left(K^{\prime}\right) \rightarrow E_{K}\left(K^{\prime}\right) \rightarrow Y_{K} \otimes_{\mathbb{Z}} X_{K}\left(K^{\prime}\right) \rightarrow H_{\mathrm{fl}}^{1}\left(\operatorname{Spec} K^{\prime}, \mathbb{G}_{\mathrm{m}}\right),
$$

respectively. Clearly, we have

$$
H_{\mathrm{fl}}^{1}\left(S^{\prime}, \mathbb{G}_{\mathrm{m}}\right)=H_{\mathrm{èt}}^{1}\left(S^{\prime}, \mathbb{G}_{\mathrm{m}}\right)=0
$$

and

$$
H_{\mathrm{fl}}^{1}\left(\operatorname{Spec} K^{\prime}, \mathbb{G}_{\mathrm{m}}\right)=H_{\text {ét }}^{1}\left(\operatorname{Spec} K^{\prime}, \mathbb{G}_{\mathrm{m}}\right)=0 .
$$

The short exact sequence $0 \rightarrow \mathbb{G}_{\mathrm{m}} \rightarrow \mathbb{G}_{\mathrm{m}, \log } \rightarrow\left(\mathbb{G}_{\mathrm{m}, \log } / \mathbb{G}_{\mathrm{m}}\right)_{S_{\mathrm{fl}}} \rightarrow 0$ gives rise to an exact sequence

$$
H_{\mathrm{fl}}^{1}\left(S^{\prime}, \mathbb{G}_{\mathrm{m}}\right) \rightarrow H_{\mathrm{fl}}^{1}\left(S^{\prime}, \mathbb{G}_{\mathrm{m}, \log }\right) \rightarrow H_{\mathrm{fl}}^{1}\left(S^{\prime},\left(\mathbb{G}_{\mathrm{m}, \log } / \mathbb{G}_{\mathrm{m}}\right)_{S_{\mathrm{fl}}}\right)
$$

Let $i^{\prime}$ denote the inclusion of the closed point $s^{\prime}$ of $S^{\prime}$ into itself. Then we get

$$
H_{\mathrm{fl}}^{1}\left(S^{\prime},\left(\mathbb{G}_{\mathrm{m}, \log } / \mathbb{G}_{\mathrm{m}}\right)_{S_{\mathrm{fl}}}\right)=H_{\mathrm{fl}}^{1}\left(S^{\prime}, i_{*}^{\prime} \mathbb{Z}\right)=H_{\mathrm{fl}}^{1}\left(s^{\prime}, \mathbb{Z}\right)=H_{\mathrm{ett}}^{1}\left(s^{\prime}, \mathbb{Z}\right)=0 .
$$

It follows that $H_{\mathrm{fl}}^{1}\left(S^{\prime}, \mathbb{G}_{\mathrm{m}, \log }\right)=0$. By Kato’s logarithmic Hilbert 90 (see [15, Thm. 3.20]), we get

$$
H_{\mathrm{kfl}}^{1}\left(S^{\prime}, \mathbb{G}_{\mathrm{m}, \log }\right)=H_{\mathrm{fl}}^{1}\left(S^{\prime}, \mathbb{G}_{\mathrm{m}, \log }\right)=0 .
$$

The exact sequences (3.8), (3.9) and (3.10) fit into the following commutative diagram

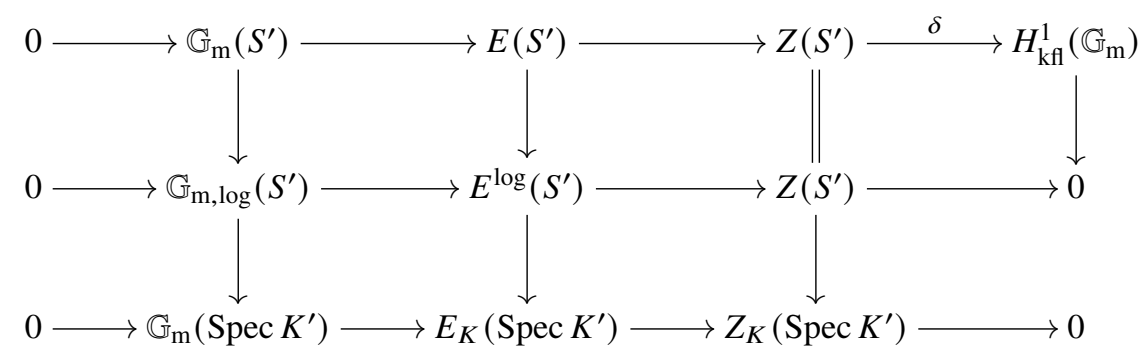


with exact rows, ${ }^{4}$ where $H_{\mathrm{kff}}^{1}\left(\mathbb{G}_{\mathrm{m}}\right)$ (respectively $Z$, respectively $Z_{K}$ ) denotes $H_{\mathrm{kfl}}^{1}\left(S^{\prime}, \mathbb{G}_{\mathrm{m}}\right)$ (respectively $Y \otimes_{\mathbb{Z}} X$, respectively $Y_{K} \otimes_{\mathbb{Z}} X_{K}$ ). Because $Y$ and $X$ become constant over $S^{\prime}$, the map

$$
Z\left(S^{\prime}\right) \rightarrow Z_{K}\left(\operatorname{Spec} K^{\prime}\right)
$$

is an isomorphism. The map $\mathbb{G}_{\mathrm{m}, \log }\left(S^{\prime}\right) \rightarrow \mathbb{G}_{\mathrm{m}}\left(\operatorname{Spec} K^{\prime}\right)$ is also an isomorphism. Therefore, the restriction map

$$
E^{\log }\left(S^{\prime}\right) \rightarrow E_{K}\left(\operatorname{Spec} K^{\prime}\right)=E^{\log }\left(\operatorname{Spec} K^{\prime}\right)
$$

is an isomorphism. We regard $E_{K}$ as an extension of $Y_{K} \otimes_{\mathbb{Z}} X_{K}$ by $\mathbb{G}_{\mathrm{m}}$, then the section $t_{K}$ (see (3.6)) of $E_{K}$ induces a section to the surjection $E^{\log }\left(S^{\prime}\right) \rightarrow Y \otimes_{\mathbb{Z}} X\left(S^{\prime}\right)$. This induced section is clearly $\operatorname{Gal}\left(S^{\prime} / S\right)$-equivariant and therefore gives rise to a section

$$
t^{\log }: Y \otimes_{\mathbb{Z}} X \rightarrow E^{\log }
$$

to the extension $E^{\log }$ of $Y \otimes_{\mathbb{Z}} X$ by $\mathbb{G}_{\mathrm{m}} \log$. The homomorphism $t^{\log }$ is automatically a section to the corresponding biextension $E^{\log }$ of $(Y, X)$ by $\mathbb{G}_{\mathrm{m}}, \log$. Note that $E^{\log }$ is also the pull-back of $P^{\log }$ along $v \times v^{\vee}$, and $t^{\log }$ gives rise to a bilinear map $s^{\log }: Y \times_{S} X \rightarrow P^{\log }$ that extends $s_{K}$. Clearly, we have the following commutative diagram:

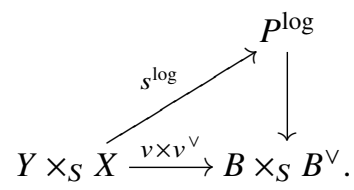

This finishes the proof.

Now we are ready to prove Theorem 3.1.

Proof of Theorem 3.1.

Step 1: We prove that $M_{K}$ extends to a két log 1-motive over $S$. Recall that $T=\mathcal{H} \operatorname{Com}_{S}\left(X, \mathbb{G}_{\mathrm{m}}\right)$, and let $T_{\log }:=\mathcal{H} \operatorname{mom}_{S}\left(X, \mathbb{G}_{\mathrm{m}, \log }\right)$. We have the following two commutative diagrams:

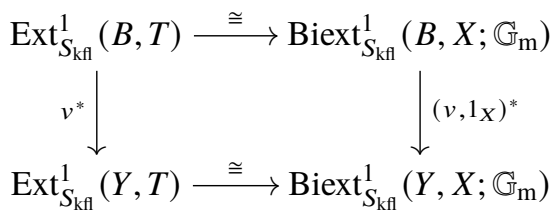

and

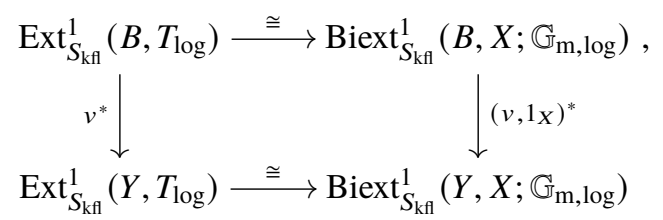

where the horizontal maps being isomorphisms comes from

$$
\mathcal{E} x t_{S_{\mathrm{kfl}}}^{1}\left(X, \mathbb{G}_{\mathrm{m}}\right)=\mathcal{E} x t_{S_{\mathrm{kfl}}}^{1}\left(X, \mathbb{G}_{\mathrm{m}, \log }\right)=0
$$

with the help of [5, Exp. VIII, §1.1.4]. Let $G \in \operatorname{Ext}_{S_{\mathrm{kfl}}}^{1}(B, T)\left(\operatorname{respectively} G_{\log } \in \operatorname{Ext}_{S_{\mathrm{kfl}}}^{1}\left(B, T_{\log }\right)\right)$ be the extension corresponding to the biextension $\left(1_{B}, v^{\vee}\right)^{*} P$ (respectively $\left(1_{B}, v^{\vee}\right)^{*} P^{\log }$ ). Then the

${ }^{4}$ We will see that the map $\delta$ is zero in Subsection 4.2. 
section $t^{\log }$ of $E^{\log }$ (see (3.12)) gives rise to a homomorphism $u^{\log }: Y \rightarrow G_{\log }$ fitting into the following commutative diagram

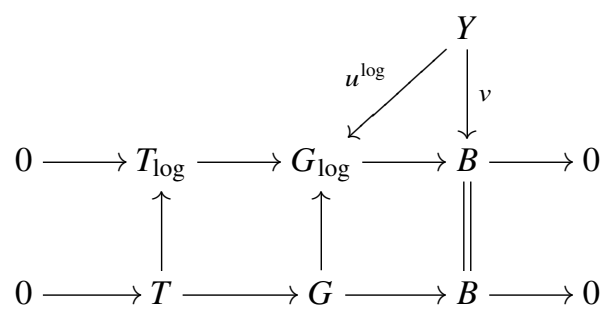

of sheaves of abelian groups on $(\mathrm{fs} / S)_{\mathrm{kfl}}$. This gives rise to a two-term complex

$$
Y \stackrel{u^{\log }}{\longrightarrow} G_{\log }
$$

Because both $X$ and $Y$ are representable by a finitely generated free abelian group over $S^{\prime}$, we have that $G \times_{S} S^{\prime}$ is an extension of the abelian scheme $B \times_{S} S^{\prime}$ by the torus $T \times_{S} S^{\prime}$ on (fs $\left./ S^{\prime}\right)$ ét by Remark 2.1 and

$$
u^{\log } \times_{S} S^{\prime}: Y \times_{S} S^{\prime} \rightarrow G_{\log } \times_{S} S^{\prime}
$$

is a $\log 1$-motive over $S^{\prime}$. Therefore, $\left[Y \stackrel{u^{\log }}{\longrightarrow} G_{\log }\right]$ is a két $\log 1$-motive over $S$. Clearly, the két $\log$ 1-motive $\left[Y \stackrel{u^{\log }}{\longrightarrow} G_{\log }\right]$ extends $M_{K}$, and its construction is functorial. Therefore, we get a functor

$$
\text { Két : TameSt-1-Mot } K \rightarrow \text { KétLog-1-Mot }{ }_{S} .
$$

Step 2: We show that

$$
\operatorname{Mor}\left(M, M^{\prime}\right) \stackrel{\cong}{\rightarrow} \operatorname{Mor}\left(M_{K}, M_{K}^{\prime}\right)
$$

for any two objects $M=\left[Y \stackrel{u}{\rightarrow} G_{\log }\right]$ and $M^{\prime}=\left[Y^{\prime} \stackrel{u^{\prime}}{\rightarrow} G_{\log }^{\prime}\right]$ in KétLog-1-Mot ${ }_{S}$, where $M_{K}:=$ $M \times_{S} \operatorname{Spec} K$ and $M_{K}^{\prime}:=M^{\prime} \times_{S}$ Spec $K$. It suffices to show that any morphism $\left(\mathfrak{f}_{-1}, \mathfrak{f}_{0}\right): M_{K} \rightarrow M_{K}^{\prime}$ extends to a unique morphism from $M$ to $M^{\prime}$. We rewrite the két $\log 1$-motive $M$ and $M^{\prime}$ as

$$
\left(Y \stackrel{v}{\rightarrow} B, X \stackrel{v^{\vee}}{\rightarrow} B^{\vee}, Y \times X \stackrel{s}{\rightarrow} P^{\log }\right)
$$

and

$$
\left(Y^{\prime} \stackrel{v^{\prime}}{\rightarrow} B^{\prime}, X^{\prime} \stackrel{v^{\prime} \vee}{\longrightarrow} B^{\prime \vee}, Y^{\prime} \times X^{\prime} \stackrel{s^{\prime}}{\rightarrow} P^{\prime} \log \right),
$$

respectively, according to (2.15). Let $v_{K}:=v \times_{S} \operatorname{Spec} K$, and similarly for $X_{K}, Y_{K}$ and so on. By [3, $\S 10.2 .12, \S 10.2 .13, \S 10.2 .14]$, the morphism $\left(\mathfrak{f}_{-1}, \mathfrak{f}_{0}\right)$ gives rise to a diagram 


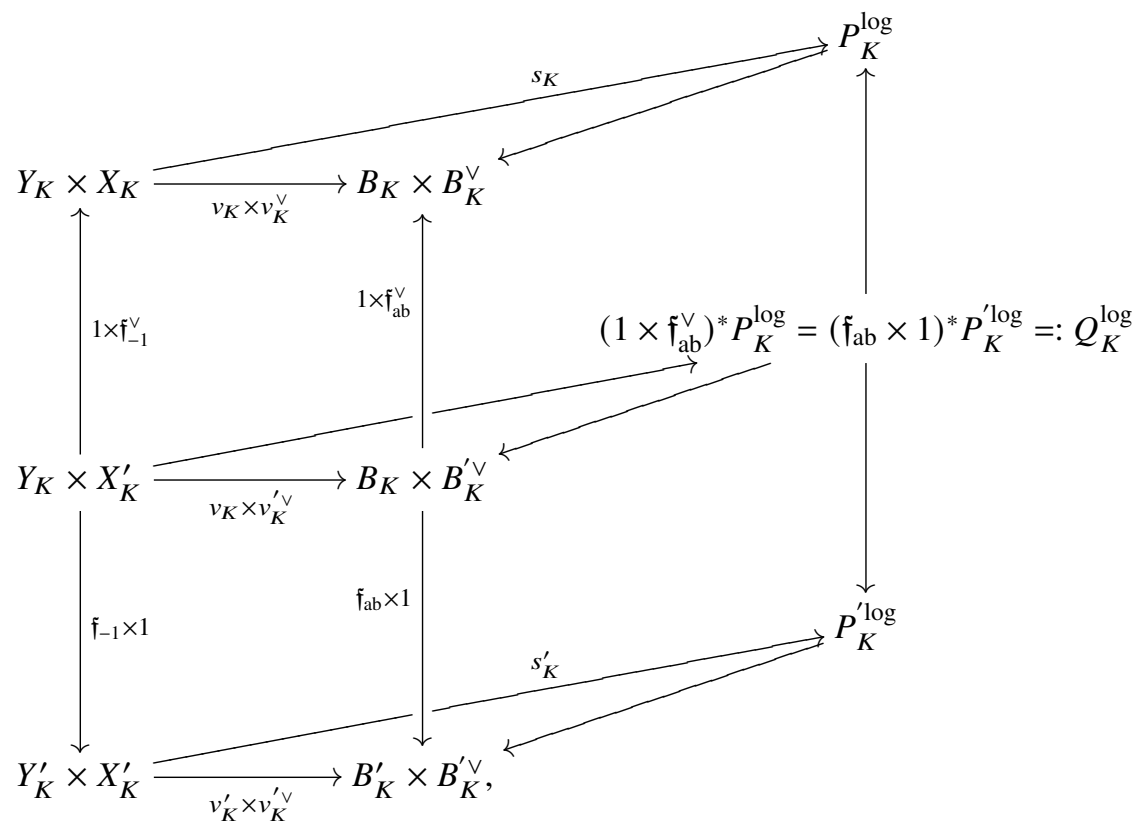

such that

(1) the two squares are commutative;

(2) for any $y \in Y_{K}$ and any $x^{\prime} \in X_{K}^{\prime}$ we have

$$
s_{K}\left(y, \mathfrak{f}_{-1}^{\vee}\left(x^{\prime}\right)\right)=s_{K}^{\prime}\left(\mathfrak{f}_{-1}(y), x^{\prime}\right)
$$

after identifying the $\mathbb{G}_{\mathrm{m}}$-torsors

$$
\left(P_{K}^{\log }\right)_{v_{K}}(y), v_{K}^{\vee}\left(\mathfrak{f}_{-1}^{\vee}\left(x^{\prime}\right)\right)
$$

and

$$
\left(P_{K}^{\prime \log }\right)_{v_{K}^{\prime}\left(\mathfrak{f}_{-1}(y)\right), v_{K}^{\prime}\left(x^{\prime}\right)}
$$

along the composition

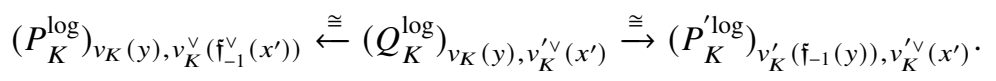

By Proposition 3.1, $\mathfrak{f}_{-1}$ (respectively $\mathfrak{f}_{-1}^{\vee}$ ) extends to a unique homomorphism $f_{-1}: Y \rightarrow Y^{\prime}$ (respectively $\left.f_{-1}^{\vee}: X^{\prime} \rightarrow X\right)$. By Theorem $3.2(1), \mathfrak{f}_{\mathrm{ab}}$ (respectively $\mathfrak{f}_{\mathrm{ab}}^{\vee}$ ) extends to a unique homomorphism $f_{\mathrm{ab}}$ : $B \rightarrow B^{\prime}$ (respectively $f_{\mathrm{ab}}^{\vee}: B^{\prime} \vee \rightarrow B^{\vee}$ ). Because $\mathfrak{f}_{\mathrm{ab}} \circ v_{K}=v_{K}^{\prime} \circ \mathfrak{f}_{-1}$, we have $f_{\mathrm{ab}} \circ v=v^{\prime} \circ f_{-1}$ by the same reason as for Lemma 3.4. Similarly, we have $f_{\mathrm{ab}}^{\vee} \circ v^{\prime} \vee=v^{\vee} \circ f_{-1}^{\vee}$. In order to show that $\left(\left(f_{-1}, f_{\mathrm{ab}}\right),\left(f_{-1}^{\vee}, f_{\mathrm{ab}}^{\vee}\right)\right)$ gives a morphism $\left(f_{-1}, f_{0}\right)$ from $M$ to $M^{\prime}$, we are left with checking the condition (2.18). But this follows from the equality (3.16) and $\Gamma\left(\operatorname{Spec} \tilde{R}, \mathbb{G}_{\mathrm{m}, \log }\right)=\Gamma\left(\operatorname{Spec} \tilde{K}, \mathbb{G}_{\mathrm{m}}\right)$ for any finite field extension $\tilde{K}$ of $K$, where $\tilde{R}$ denotes the normalisation of $R$ in $\tilde{K}$ and Spec $\tilde{R}$ is endowed with the canonical $\log$ structure. The uniqueness of $\left(f_{-1}, f_{0}\right)$ follows from those of $f_{-1}, f_{\mathrm{ab}}, f_{-1}^{\vee}$ and $f_{\mathrm{ab}}^{\vee}$.

Step 3: By Step 1 and Step 2, the functor Két is fully faithful. For any $M \in$ KétLog-1-Mot ${ }_{S}$, the base change $M_{K}$ of $M$ to Spec $K$ lies in TameSt-1-Mot ${ }_{K}$. By Step 2, the identity morphism of $M_{K}$ extends to a unique isomorphism from $M$ to $\operatorname{Két}\left(M_{K}\right)$. Therefore, Két is essentially surjective and hence an equivalence of categories. This finishes the proof of Theorem 3.1. 
Example 3.4. Let $M_{K}=\left[Y_{K} \stackrel{u_{K}}{\longrightarrow} G_{K}\right] \in$ TameSt-1-Mot ${ }_{K}$, and $G_{K}$ an extension of an abelian variety $B_{K}$ by a torus $T_{K}$ over $K$. Assume that either $Y_{K}$ is not unramified, or $T_{K}$ is not unramified or $B_{K}$ does not have good reduction. Then the két $\log 1$-motive $M^{\log }:=$ Két $\left(M_{K}\right)$ is not a log 1-motive by Example 3.1, Example 3.2 and Example 3.3.

Corollary 3.1. Let the notation and assumptions be as in Theorem 3.1. We further assume that both $Y_{K}$ and $G_{K}$ have good reduction. Then the két $\log 1$-motive $M^{\log }:=\operatorname{Két}\left(M_{K}\right)=\left[Y \stackrel{u^{\log }}{\longrightarrow} G_{\log }\right]$ associated to $M_{K}$ is a log 1-motive.

Proof. Because both $Y_{K}$ and $G_{K}$ have good reduction, both $X$ and $Y$ are étale locally representable by a finitely generated free abelian group over $S$. Therefore, $G$ is an extension of the abelian scheme $B$ by the torus $T$ on (fs $/ S)_{\mathrm{kfl}}$. By Remark 2.1, $G$ comes from an extension on (fs $\left./ S\right)_{\text {ét }}$. It follows that $\left[Y \stackrel{u^{\log }}{\longrightarrow} G_{\log }\right]$ is a $\log 1$-motive over $S$.

Remark 3.1. Corollary 3.1 shows that a $\log 1$-motive in the sense of $[13, \S 4.6 .1]$ extends uniquely to a $\log 1$-motive in our sense (i.e., in the sense of [8, Defn. 2.2]). This must have been known to Kazuya Kato, who is one of the authors of both [13] and [8].

Remark 3.2. As we have proposed in Section 1, as a generalisation of Theorem 3.1, it is natural to ask whether every strict (not just strict tamely ramified) 1-motive over $K$ extends to a unique kfl $\log$ 1-motive over $S$, where a kfl $\log 1$-motive can be defined as in Definition 2.6 by using the Kummer flat topology instead of the Kummer étale topology. In the proofs of Proposition 3.1, Proposition 3.2 and Theorem 3.2, we have used explicitly the theory of logarithmic fundamental group, which is only available in the Kummer étale topology. Therefore, our method in this article does not work for the Kummer flat case.

\section{Monodromy}

In this section, we construct a monodromy pairing for a tamely ramified strict 1-motive $M_{K}$ over a discrete valuation field via the két log 1-motive $M^{\log }$ associated to $M_{K}$. We compare our monodromy pairing with Raynaud's geometric monodromy pairing from [16, §4.3]. Note that although the base field $K$ in $[16, \S 4.3]$ is assumed to be complete, the definition of the geometric monodromy works for any discrete valuation field. We remark that Raynaud's geometric monodromy paring is defined for all strict 1 -motives over $K$, whereas our monodromy pairing is only defined for tamely ramified strict 1-motives.

\subsection{Logarithmic monodromy pairing}

We adopt the setup and notation from last section. Consider the following pushout diagram:

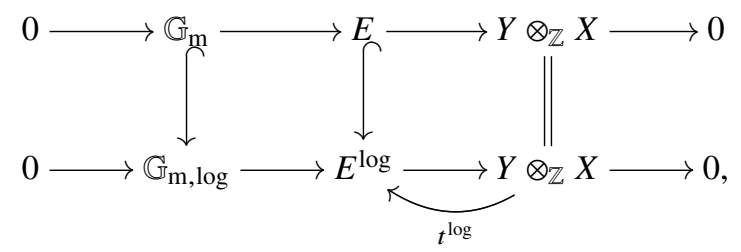

where $t^{\log }$ is the section (3.12). Then the section $t^{\log }$ induces a linear map

$$
Y \otimes_{\mathbb{Z}} X \stackrel{t^{\log }}{\longrightarrow} E^{\log } \rightarrow E^{\log } / E \cong\left(\mathbb{G}_{\mathrm{m}, \log } / \mathbb{G}_{\mathrm{m}}\right)_{S_{\mathrm{kfl}}},
$$

which corresponds to a bilinear map

$$
\langle-,-\rangle: Y \times X \rightarrow\left(\mathbb{G}_{\mathrm{m}, \log } / \mathbb{G}_{\mathrm{m}}\right)_{S_{\mathrm{kfl}}} .
$$

This pairing is nothing but the monodromy pairing (2.6) for the két log 1-motive $M^{\log }$. 
Definition 4.1. We call the pairing (4.2) the logarithmic monodromy pairing of the tamely ramified strict 1-motive $M_{K}$.

Proposition 4.1. Let the assumptions and notation be as in Theorem 3.1 and its proof. The monodromy pairing (4.2) vanishes if and only if the section $t^{\log }$ is induced from a section $t: Y \otimes_{\mathbb{Z}} X \rightarrow E$ of $E$.

When such a section $t$ exists, it corresponds to a section $s: Y \times_{S} X \rightarrow P$, which further corresponds to a map $u: Y \rightarrow G$. The maps $s$ and $u$ extend the diagrams (3.13) and (3.14) to the commutative diagrams

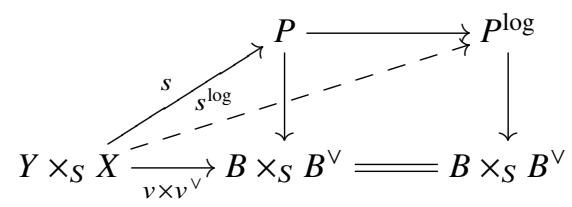

and

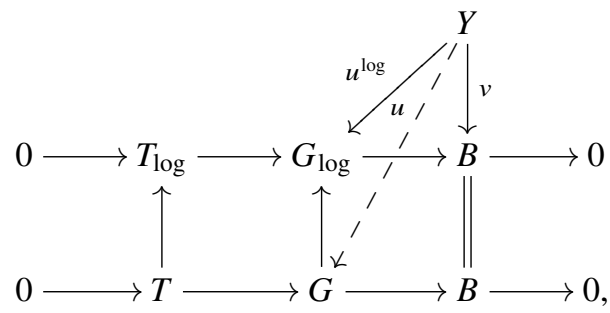

respectively. Therefore, the given 1-motive $M_{K}$ extends to a unique két 1-motive $M=[Y \stackrel{u}{\rightarrow} G]$ such that the két log 1-motive $M^{\log }$ associated to $M_{K}$ is induced from $M$.

Proof. By the construction of the monodromy pairing, its vanishing is clearly equivalent to $t^{\log }$ being induced from a section $t: Y \otimes_{\mathbb{Z}} X \rightarrow E$ of $E$. The proof of the rest is similar to the proof of Theorem 3.1.

Proposition 4.2. Let $M_{K}$ be a tamely ramified strict 1-motive over $K$ and $M^{\log }=\left[Y \stackrel{u^{\log }}{\longrightarrow} G_{\log }\right]$ the két $\log 1$-motive associated to $M_{K}$. Assume that the logarithmic monodromy pairing of $M_{K}$ is induced by a pairing $\mu_{\pi}: Y \times X \rightarrow \pi^{\mathbb{Z}}$. Let

$$
u_{2, \pi}^{\log }: Y \rightarrow T_{\log }=\mathcal{H}^{\circ} m_{S_{\mathrm{kff}}}\left(X, \mathbb{G}_{\mathrm{m}, \log }\right) \subset G_{\log }
$$

be the map induced by $\mu_{\pi}$, and $u_{1, \pi}^{\log }:=u^{\log }-u_{2, \pi}^{\log }$. Then $u_{1, \pi}^{\log }$ factors as

$$
Y \stackrel{u_{1, \pi}}{\longrightarrow} G \hookrightarrow G_{\log }
$$

that is, the két log 1-motive $\left[Y \stackrel{u_{1, \pi}^{\log }}{\longrightarrow} G_{\log }\right]$ is induced from the két 1-motive $\left[Y \stackrel{u_{1, \pi}}{\longrightarrow} G\right]$.

Proof. It suffices to prove that $u_{1, \pi}^{\log }$ factors through $G \hookrightarrow G_{\log }$; the rest is clear. The monodromy pairing of the két log 1-motive $\left[Y \stackrel{u_{1, \pi}^{\log }}{\longrightarrow} G_{\log }\right]$ is the difference of the monodromy pairings of $\left[Y \stackrel{u^{\log }}{\longrightarrow} G_{\log }\right]$ and $\left[Y \stackrel{u_{2, \pi}^{\log }}{\longrightarrow} G_{\log }\right]$. Because the two monodromy pairings agree, the monodromy pairing of $\left[Y \stackrel{u_{1, \pi}^{\log }}{\longrightarrow} G_{\log }\right]$ vanishes. By Proposition 4.1, we are done. 
Example 4.1. Let $M_{K}=\left[Y_{K} \stackrel{u_{K}}{\longrightarrow} G_{K}\right]$ be a tamely ramified strict 1-motive over $K$. Assume that both $Y_{K}$ and $G_{K}$ have good reduction. Then both $Y$ and $X$ are étale locally constant. Therefore, the monodromy pairing

$$
\langle-,-\rangle: Y \times X \rightarrow\left(\mathbb{G}_{\mathrm{m}, \log } / \mathbb{G}_{\mathrm{m}}\right)_{S_{\mathrm{kfl}}}
$$

factors through the canonical homomorphism

$$
\pi^{\mathbb{Z}} \cong M_{S}^{\mathrm{gp}} / \mathcal{O}_{S}^{\times} \rightarrow\left(\mathbb{G}_{\mathrm{m}, \log } / \mathbb{G}_{\mathrm{m}}\right)_{S_{\mathrm{kfl}}}
$$

In other words, the monodromy pairing of $M_{K}$ satisfies the assumption of Proposition 4.2 in this case.

Proposition 4.2 gives rise to a decomposition $u^{\log }=u_{1, \pi}^{\log }+u_{2, \pi}^{\log }$ whose construction involves the chosen uniformiser $\pi$. Next we look for a decomposition $u^{\log }=u_{1}^{\log }+u_{2}^{\log }$ that is independent of the choice of a uniformiser and satisfies the following condition:

$$
u_{1}^{\log } \text { is induced by some map } u_{1}: Y \rightarrow G \text { and } u_{2}^{\log } \text { factors through } T_{\log } \hookrightarrow G_{\log } \text {. }
$$

Proposition 4.3. Let $M_{K}$ be a tamely ramified strict 1-motive over $K$ and $M^{\log }=\left[Y \stackrel{u^{\log }}{\longrightarrow} G_{\log }\right]$ the két log 1-motive associated to $M_{K}$. The decompositions $u^{\log }=u_{1}^{\log }+u_{2}^{\log }$ satisfying the condition (4.5) correspond canonically to the trivialisations $t: Y \otimes_{\mathbb{Z}} X \rightarrow E$ of the extension $E$ from (4.1). And under this correspondence, the homomorphism $u_{2}^{\log }$ is induced by the difference homomorphism $t^{\log }-t$, where $t^{\log }$ is as in (4.1).

Proof. Given a decomposition $u^{\log }=u_{1}^{\log }+u_{2}^{\log }$ satisfying the condition (4.5), the map $u_{1}$ associated to $u_{1}^{\log }$ gives rise to a section $t: Y \otimes_{\mathbb{Z}} X \rightarrow E$ of $E$.

Conversely, given a section $t: Y \otimes_{\mathbb{Z}} X \rightarrow E$ of $E$, the decomposition

$$
t^{\log }=t+\left(t^{\log }-t\right):=t_{1}+t_{2}
$$

gives rise to a decomposition $u^{\log }=u_{1}^{\log }+u_{2}^{\log }$ with $u_{i}^{\log }$ induced by $t_{i}$. It is clear that $u_{1}^{\log }$ factors through $G \hookrightarrow G_{\log }$. By an easy calculation $t^{\log }-t$ factors through $\mathbb{G}_{\mathrm{m}, \log } \hookrightarrow E^{\log }$; therefore, $u_{2}^{\log }$ factors as $Y \rightarrow T_{\log } \rightarrow G_{\log }$. Hence, the decomposition $u^{\log }=u_{1}^{\log }+u_{2}^{\log }$ satisfies the condition (4.5).

As before, let $Z:=Y \otimes_{\mathbb{Z}} X$. We abbreviate $\left(\mathbb{G}_{\mathrm{m}, \log } / \mathbb{G}_{\mathrm{m}}\right)_{S_{\mathrm{kfl}}}$ as $\overline{\mathbb{G}}_{\mathrm{m}, \log }$. Applying the functor Hom $_{S_{\mathrm{kfl}}}(Z,-)$ to the short exact sequence

$$
0 \rightarrow \mathbb{G}_{\mathrm{m}} \rightarrow \mathbb{G}_{\mathrm{m}, \log } \rightarrow \overline{\mathbb{G}}_{\mathrm{m}, \log } \rightarrow 0
$$

we get an exact sequence

$$
\operatorname{Hom}_{S_{\mathrm{kfl}}}\left(Z, \mathbb{G}_{\mathrm{m}, \log }\right) \stackrel{\alpha}{\rightarrow} \operatorname{Hom}_{S_{\mathrm{kfl}}}\left(Z, \overline{\mathbb{G}}_{\mathrm{m}, \log }\right) \rightarrow \operatorname{Ext}_{S_{\mathrm{kfl}}}^{1}\left(Z, \mathbb{G}_{\mathrm{m}}\right) \rightarrow \operatorname{Ext}_{S_{\mathrm{kfl}}}^{1}\left(Z, \mathbb{G}_{\mathrm{m}, \log }\right)
$$

Let $\mu^{\log } \in \operatorname{Hom}_{S_{\mathrm{kfl}}}\left(Z, \overline{\mathbb{G}}_{\mathrm{m}, \log }\right)$ be the element corresponding to the logarithmic monodromy pairing $\langle-,-\rangle$ of $M_{K}$. Then the element $E$ of $\operatorname{Ext}_{S_{\mathrm{kfl}}}^{1}\left(Z, \mathbb{G}_{\mathrm{m}}\right)$ is the image of $\mu^{\log }$ along the map $\operatorname{Hom}_{S_{\mathrm{kfl}}}\left(Z, \overline{\mathbb{G}}_{\mathrm{m}, \log }\right) \rightarrow \operatorname{Ext}_{S_{\mathrm{kfl}}}^{1}\left(Z, \mathbb{G}_{\mathrm{m}}\right)$. If $E$ is trivial, then the subset

$$
\alpha^{-1}\left(\mu^{\log }\right) \subset \operatorname{Hom}_{S_{\mathrm{kfl}}}\left(Z, \mathbb{G}_{\mathrm{m}, \log }\right)=\operatorname{Hom}_{S_{\mathrm{kfl}}}\left(Y, T_{\log }\right)
$$

is not empty, and its elements correspond to the choices of $u_{2}^{\log }$. 


\subsection{Comparison with Raynaud's geometric monodromy}

Because $B$ and $B^{\vee}$ become abelian schemes after base change to $S^{\prime}, P \times_{S} S^{\prime}$ is the Poincaré biextension of the abelian schemes $B \times{ }_{S} S^{\prime}$ and $B^{\vee} \times_{S} S^{\prime}$; in particular,

$$
P \times_{S} S^{\prime} \in \operatorname{Biext}_{S_{\mathrm{fl}}^{\prime}}^{1}\left(B \times_{S} S^{\prime}, B^{\vee} \times_{S} S^{\prime} ; \mathbb{G}_{\mathrm{m}}\right) .
$$

It follows that the extension $E \times_{S} S^{\prime}$ lies in the subgroup $\operatorname{Ext}_{S_{\mathrm{fl}}^{\prime}}^{1}\left(\left(Y \otimes_{\mathbb{Z}} X\right) \times_{S} S^{\prime}, \mathbb{G}_{\mathrm{m}}\right)$ of the group $\operatorname{Ext}_{S_{\mathrm{kff}}^{\prime}}^{1}\left(\left(Y \otimes_{\mathbb{Z}} X\right) \times_{S} S^{\prime}, \mathbb{G}_{\mathrm{m}}\right)$. Therefore, the image of the map $\delta$ from (3.11) lands in the subgroup $H_{\mathrm{fl}}^{1}\left(S^{\prime}, \mathbb{G}_{\mathrm{m}}\right)$ of $H_{\mathrm{kfl}}^{1}\left(S^{\prime}, \mathbb{G}_{\mathrm{m}}\right)$. Because $H_{\mathrm{fl}}^{1}\left(S^{\prime}, \mathbb{G}_{\mathrm{m}}\right)=0$, the diagram (3.11) gives rise to the following commutative diagram

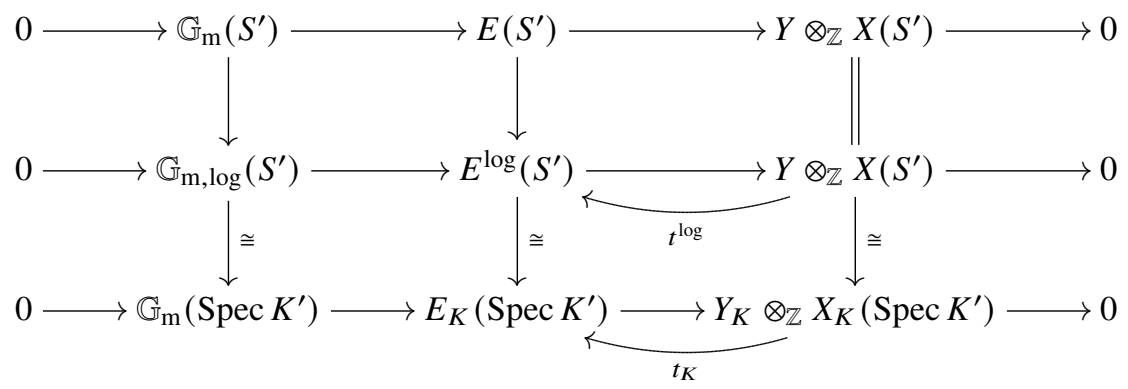

with exact rows. Then the pairing (4.2) induces a pairing

$$
Y\left(S^{\prime}\right) \times X\left(S^{\prime}\right) \rightarrow \Gamma\left(S^{\prime},\left(\mathbb{G}_{\mathrm{m}, \log } / \mathbb{G}_{\mathrm{m}}\right)_{S_{\mathrm{kff}}}\right),
$$

which actually factorises through a pairing

$$
\langle-,-\rangle: Y\left(S^{\prime}\right) \times X\left(S^{\prime}\right) \rightarrow \mathbb{G}_{\mathrm{m}, \log }\left(S^{\prime}\right) / \mathbb{G}_{\mathrm{m}}\left(S^{\prime}\right)
$$

by the diagram (4.6). The pairing (4.7) is clearly $\mathrm{Gal}\left(S^{\prime} / S\right)$-equivariant and also determines the monodromy pairing (4.2). We have the canonical identification $\mathbb{G}_{\mathrm{m}, \log }\left(S^{\prime}\right) / \mathbb{G}_{\mathrm{m}}\left(S^{\prime}\right)=\left(K^{\prime}\right)^{\times} /\left(R^{\prime}\right)^{\times}$. Let $\bar{K}$ be a separable closure of $K$ containing $K^{\prime}$. Then the valuation of $K$ extends to a unique valuation of $\bar{K}$ taking value in $\mathbb{Q}$. Applying the valuation of $\bar{K}$ on $\left(K^{\prime}\right)^{\times} /\left(R^{\prime}\right)^{\times}$, we get a monomorphism $\mathbb{G}_{\mathrm{m}, \log }\left(S^{\prime}\right) / \mathbb{G}_{\mathrm{m}}\left(S^{\prime}\right)=\left(K^{\prime}\right)^{\times} /\left(R^{\prime}\right)^{\times} \hookrightarrow \mathbb{Q}$. Then the pairing (4.7) induces a pairing

$$
\langle-,-\rangle: Y\left(S^{\prime}\right) \times X\left(S^{\prime}\right) \rightarrow \mathbb{Q}
$$

that is equivariant with respect to the action of $\operatorname{Gal}\left(S^{\prime} / S\right)=\operatorname{Gal}\left(K^{\prime} / K\right)$.

Proposition 4.4. The pairing (4.8) coincides with the geometric monodromy pairing $\mu: Y_{K}\left(\operatorname{Spec} K^{\prime}\right) \times$ $X_{K}\left(\operatorname{Spec} K^{\prime}\right) \rightarrow \mathbb{Q}$ from [16, \$4.3].

Proof. The map $t_{K}$ in the diagram (4.6) induces a homomorphism

$$
\begin{aligned}
Y_{K} \otimes_{\mathbb{Z}} X_{K}\left(\operatorname{Spec} K^{\prime}\right) \rightarrow E_{K}\left(\operatorname{Spec} K^{\prime}\right) / E\left(S^{\prime}\right) & \cong \mathbb{G}_{\mathrm{m}}\left(\operatorname{Spec} K^{\prime}\right) / \mathbb{G}_{\mathrm{m}}\left(S^{\prime}\right) \\
& =\left(K^{\prime}\right)^{\times} /\left(R^{\prime}\right)^{\times}
\end{aligned}
$$

that gives rise to exactly the monodromy pairing from [16, §4.3] after applying the unique valuation of $\bar{K}$, which extends the valuation on $K$. Because the second row and the third row in the diagram (4.6) are isomorphic, we are done. 
If Raynaud's geometric monodromy pairing $\mu$ factors through $\mathbb{Z} \hookrightarrow \mathbb{Q},[16$, Prop. 4.5.1] gives a decomposition $u_{K}=u_{K, \pi}^{1}+u_{K, \pi}^{2}$ such that

the $K$-1-motive $M_{K, \pi}^{1}=\left[Y_{K} \stackrel{u_{K, \pi}^{1}}{\longrightarrow} G_{K}\right]$ has potentially good reduction;

and $u_{K, \pi}^{2}$ factors through the torus part $T_{K}$ of $G_{K}$.

When the field $K$ is complete, such a decomposition is made independent of the choice of the uniformiser $\pi$ in [16, Prop. 4.5.3] via rigid geometry. And a decomposition $u_{K}=u_{K}^{1}+u_{K}^{2}$ that satisfies the condition analogous to (4.9) and is independent of the choice of a uniformiser corresponds to a trivialisation $\tau: Z_{K}=Y_{K} \otimes_{\mathbb{Z}} X_{K} \rightarrow \mathcal{E}_{\text {rig }}$ of the extension $\mathcal{E}_{\text {rig }}$ of $Z_{K}$ by $U_{\text {rig }}$ defined in [16, Rmk. 4.5.2 (iii)].

Lemma 4.1. Raynaud's monodromy pairing factors through $\mathbb{Z} \hookrightarrow \mathbb{Q}$ if and only if the assumption of Proposition 4.2 holds; that is, the logarithmic monodromy pairing (4.2) of $M_{K}$ is induced by a pairing $\mu_{\pi}: Y \times X \rightarrow \pi^{\mathbb{Z}}$.

Proof. If the logarithmic monodromy is induced by $\mu_{\pi}$, then the image of the composition

$$
Y \otimes_{\mathbb{Z}} X\left(S^{\prime}\right) \stackrel{t^{\log }}{\longrightarrow} E^{\log }\left(S^{\prime}\right) / E\left(S^{\prime}\right) \cong \mathbb{G}_{\mathrm{m}, \log }\left(S^{\prime}\right) / \mathbb{G}_{\mathrm{m}}\left(S^{\prime}\right)
$$

lands in $\pi^{\mathbb{Z}_{\mathbb{G}_{\mathrm{m}}}}\left(S^{\prime}\right) / \mathbb{G}_{\mathrm{m}}\left(S^{\prime}\right)$. Because $t^{\log }$ restricts to $t_{K}$, the image of the composition

$$
\begin{aligned}
Y_{K} \otimes_{\mathbb{Z}} X_{K}\left(\operatorname{Spec} K^{\prime}\right) \rightarrow E_{K}\left(\operatorname{Spec} K^{\prime}\right) / E\left(S^{\prime}\right) & \cong \mathbb{G}_{\mathrm{m}}\left(\operatorname{Spec} K^{\prime}\right) / \mathbb{G}_{\mathrm{m}}\left(S^{\prime}\right) \\
& =\left(K^{\prime}\right)^{\times} /\left(R^{\prime}\right)^{\times}
\end{aligned}
$$

lands in $\pi^{\mathbb{Z}}\left(R^{\prime}\right)^{\times} /\left(R^{\prime}\right)^{\times}$. Thus, Raynaud's monodromy factors through $\mathbb{Z} \hookrightarrow \mathbb{Q}$.

Conversely, if Raynaud's monodromy factors through $\mathbb{Z} \hookrightarrow \mathbb{Q}$, we clearly have that the image of the composition (4.10) lands in $\pi^{\mathbb{Z}} \mathbb{G}_{\mathrm{m}}\left(S^{\prime}\right) / \mathbb{G}_{\mathrm{m}}\left(S^{\prime}\right) \cong \pi^{\mathbb{Z}}$. Because the logarithmic monodromy pairing is induced by the pairing (4.7), we are done.

Under the assumption that Raynaud's monodromy factors through $\mathbb{Z} \hookrightarrow \mathbb{Q}$, we have Raynaud's decomposition $u_{K}=u_{K, \pi}^{1}+u_{K, \pi}^{2}$, as well as the decompositions $u^{\log }=u_{1, \pi}^{\log }+u_{2, \pi}^{\log }$ and $u^{\log }=u_{1}^{\log }+u_{2}^{\log }$ from Proposition 4.2 by Lemma 4.1. If $K$ is complete, we also have Raynaud's decomposition $u_{K}=$ $u_{K}^{1}+u_{K}^{2}$. From the constructions of these decompositions, it is easy to check the following proposition.

Proposition 4.5. The restriction of the decomposition $u^{\log }=u_{1, \pi}^{\log }+u_{2, \pi}^{\log }$ from $S$ to Spec $K$ gives rise to Raynaud's decomposition $u_{K}=u_{K, \pi}^{1}+u_{K, \pi}^{2}$. If $K$ is complete, the decomposition $u^{\log }=u_{1}^{\log }+u_{2}^{\log }$ restricts to $u_{K}=u_{K}^{1}+u_{K}^{2}$.

\section{Log finite group objects associated to két log 1-motives}

\subsection{Log finite group objects}

Let $S$ be a locally noetherian fs log scheme. Kato has developed a theory of log finite group objects that is parallel to the theory of finite flat group schemes in the non-log world. The main references are [12] and [14].

Definition 5.1. The category (fin $/ S)_{\mathrm{c}}$ is the full subcategory of the category of sheaves of finite abelian groups over $(\mathrm{fs} / S)_{\mathrm{kfl}}$ consisting of objects that are representable by a classical finite flat group scheme over $S$. Here 'classical' means that the log structure of the representing log scheme is the one induced from $S$.

The category $(\mathrm{fin} / S)_{\mathrm{f}}$ is the full subcategory of the category of sheaves of finite abelian groups over $(\mathrm{fs} / S)_{\mathrm{kfl}}$ consisting of objects that are representable by a classical finite flat group scheme over a Kummer 
flat cover of $S$. For $F \in(\text { fin } / S)_{\text {f }}$, let $U \rightarrow S$ be a log flat cover of $S$ such that $F_{U}:=F \times_{S} U \in(\text { fin } / U)_{\mathrm{c}}$. Then the rank of $F$ is defined to be the rank of $F_{U}$ over $U$.

The category $(\mathrm{fin} / S)_{\mathrm{r}}$ is the full subcategory of $(\mathrm{fin} / S)_{\mathrm{f}}$ consisting of objects that are representable by a $\log$ scheme over $S$.

The category (fin $/ S)_{\text {é }}$ is the full subcategory of (fin $\left./ S\right)_{\mathrm{f}}$ consisting of objects $F$ such that there exists a Kummer étale cover $U$ of $S$ such that $F \times_{S} U \in(\text { fin } / U)_{\text {r }}$.

Let $F \in(\mathrm{fin} / S)_{\mathrm{f}}$. Then the Cartier dual of $F$ is defined to be the sheaf

$$
F^{*}:=\mathcal{H}^{\circ o m} S_{\mathrm{kfl}}\left(F, \mathbb{G}_{\mathrm{m}}\right) .
$$

By the definition of $(\mathrm{fin} / S)_{\mathrm{f}}$, it is clear that $F^{*} \in(\mathrm{fin} / S)_{\mathrm{f}}$.

The category $(\mathrm{fin} / S)_{\mathrm{d}}$ is the full subcategory of $(\mathrm{fin} / S)_{\mathrm{r}}$ consisting of objects whose Cartier dual also lies in $(\mathrm{fin} / S)_{\mathrm{r}}$.

Proposition 5.1 (Kato). The categories $(\mathrm{fin} / S)_{\mathrm{f}}$, $(\mathrm{fin} / S)_{\mathrm{e}},(\mathrm{fin} / S)_{\mathrm{r}}$ and $(\mathrm{fin} / S)_{\mathrm{d}}$ are closed under extensions in the category of sheaves of abelian groups on $(\mathrm{fs} / S)_{\mathrm{kfl}}$.

Proof. See [12, Prop. 2.3].

Definition 5.2. Let $p$ be a prime number. A $\log p$-divisible group (respectively két $\log p$-divisible group, respectively kfl $\log p$-divisible group) over $S$ is a sheaf of abelian groups $G$ on (fs $/ S)_{\text {kfl }}$ satisfying the following:

(1) $G=\bigcup_{n \geq 0} G_{n}$ with $G_{n}:=\operatorname{ker}\left(p^{n}: G \rightarrow G\right)$;

(2) $p: G \rightarrow G$ is surjective;

(3) $G_{n} \in(\text { fin } / S)_{\mathrm{r}}\left(\right.$ respectively $G_{n} \in(\text { fin } / S)_{\text {é }}$, respectively $\left.G_{n} \in(\text { fin } / S)_{\mathrm{f}}\right)$ for any $n>0$.

We denote by $(p-\operatorname{div} / S)^{\log }$ (respectively $(p-\operatorname{div} / S)_{\mathrm{e}}^{\log }$, respectively $\left.(p-\operatorname{div} / S)_{\mathrm{f}}^{\log }\right)$ the category of $\log$ $p$-divisible groups (respectively két $\log p$-divisible groups, respectively kfl $\log p$-divisible groups) over $S$. The full subcategory of $(p \text {-div } / S)^{\log }$ consisting of objects $G$ with $G_{n} \in(\text { fin } / S)_{\mathrm{d}}$ for all $n>0$ will be denoted by $(p \text {-div } / S)_{\mathrm{d}}^{\log }$. A $\log p$-divisible group $G$ with $G_{n} \in(\text { fin } / S)_{\mathrm{c}}$ for all $n>0$ is clearly just a classical $p$-divisible group, and we denote the full subcategory of $(p \text {-div } / S)_{\mathrm{d}}^{\log }$ consisting of classical $p$-divisible groups by $(p-\operatorname{div} / S)$.

Remark 5.1. For $G \in(p-\operatorname{div} / S)^{\log }$ to lie in $(p-\operatorname{div} / S)_{\mathrm{d}}^{\log }$, it is enough to require $G_{1} \in(\mathrm{fin} / S)_{\mathrm{d}}$. We explain this as follows. The short exact sequence $0 \rightarrow G_{1} \rightarrow G_{2} \rightarrow G_{1} \rightarrow 0$ gives an exact sequence

$$
0 \rightarrow G_{1}^{*} \rightarrow G_{2}^{*} \stackrel{\alpha}{\rightarrow} G_{1}^{*} \rightarrow \mathcal{E} x t_{S_{\mathrm{fl}}}^{1}\left(G_{1}, \mathbb{G}_{\mathrm{m}}\right)
$$

We claim that $\alpha$ is an epimorphism for the Kummer flat topology. To prove the claim, we may assume that $G_{1}$ is a classical finite flat group scheme. Then we have $\mathcal{E} x t_{S_{\mathrm{ff}}}^{1}\left(G_{1}, \mathbb{G}_{\mathrm{m}}\right)=0$ by [5, Exp. VIII, Prop. 3.3.1] and $R^{1} m_{*} \mathbb{G}_{\mathrm{m}}=0$, where $m$ denotes the natural map from the classical flat site to the classical étale site. Therefore, we get a short exact sequence

$$
0 \rightarrow G_{1}^{*} \rightarrow G_{2}^{*} \rightarrow G_{1}^{*} \rightarrow 0
$$

of sheaves of abelian groups on $(\mathrm{fs} / S)_{\mathrm{kfl}}$. Thus, $G_{2}^{*} \in(\mathrm{fin} / S)_{\mathrm{r}}$ by [12, Prop. 2.3]. Inductively we get $G_{n}^{*} \in(\text { fin } / S)_{\text {r }}$ for all $n>1$.

\subsection{Log finite group objects associated to két log 1-motives}

Definition 5.3. Let $S$ be an fs $\log$ scheme, $M^{\log }=\left[Y \stackrel{u}{\rightarrow} G_{\log }\right]$ a két $\log 1$-motive over $S$ and $n$ a positive integer. By Lemma 2.2 and Corollary 2.1, we can regard $M^{\log }$ as a complex of sheaves on $(\mathrm{fs} / S)_{\mathrm{kfl}}$ and 
define

$$
T_{\mathbb{Z} / n \mathbb{Z}}\left(M^{\log }\right):=H^{-1}\left(M^{\log } \otimes_{\mathbb{Z}}^{\mathrm{L}} \mathbb{Z} / n \mathbb{Z}\right) .
$$

Proposition 5.2. Let $S$ be a locally noetherian $f$ s log scheme,

$$
M^{\log }=\left[Y \stackrel{u}{\rightarrow} G_{\log }\right]
$$

a két log 1-motive over $S$ and $n$ a positive integer. Then we have the following:

(1) $T_{\mathbb{Z} / n \mathbb{Z}}\left(M^{\log }\right)$ fits into the following exact sequence

$$
0 \rightarrow G_{\log }[n] \rightarrow T_{\mathbb{Z} / n \mathbb{Z}}\left(M^{\log }\right) \rightarrow Y / n Y \rightarrow 0
$$

of sheaves of abelian groups on $(\mathrm{fs} / S)_{\mathrm{kfl}}$, where $G_{\log }[n]$ denotes the $n$-torsion subgroup sheaf of $G_{\log }$.

(2) $T_{\mathbb{Z} / n \mathbb{Z}}\left(M^{\log }\right) \in(\mathrm{fin} / S)_{\text {é }}$

(3) Let $m$ be another positive integer. Then the map

$$
T_{\mathbb{Z} / m n \mathbb{Z}}\left(M^{\log }\right) \rightarrow T_{\mathbb{Z} / n \mathbb{Z}}\left(M^{\log }\right)
$$

induced by $\mathbb{Z} / m n \mathbb{Z} \stackrel{\bmod m}{\longrightarrow} \mathbb{Z} / n \mathbb{Z}$ is surjective.

(4) If $M^{\log }$ is a log 1-motive, then $T_{\mathbb{Z} / n \mathbb{Z}}\left(M^{\log }\right) \in(\mathrm{fin} / S)_{\mathrm{d}}$.

Proof. For part (1), by [16, §3.1], it suffices to show that the multiplication by $n$ is injective on $Y$ and surjective on $G_{\log }$ for the Kummer flat topology. The injectivity of the map $Y \stackrel{n}{\rightarrow} Y$ is trivial. We are reduced to show the surjectivity of the map $G_{\log } \stackrel{n}{\rightarrow} G_{\log }$. Without loss of generality, we may assume that $M^{\log }$ is a $\log 1$-motive. Let $G$ be an extension of an abelian scheme $B$ by a torus $T$ over $S$. Consider the following commutative diagram

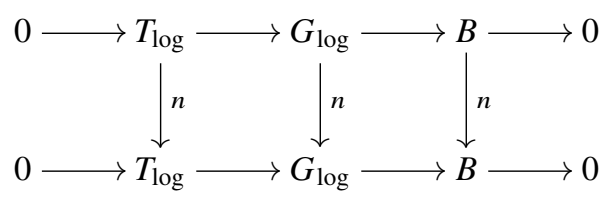

with exact rows. The multiplication by $n$ is clearly surjective on $B$, and the surjectivity of the multiplication by $n$ on $T_{\log }$ follows from the surjectivity of $\mathbb{G}_{\mathrm{m}, \log } \stackrel{n}{\rightarrow} \mathbb{G}_{\mathrm{m}, \log }$. It follows that $G_{\log } \stackrel{n}{\rightarrow} G_{\log }$ is surjective.

For part (2), we may still assume that $M^{\log }$ is a $\log 1$-motive. We have a short exact sequence $0 \rightarrow T_{\log }[n] \rightarrow G_{\log }[n] \rightarrow B[n] \rightarrow 0$. Let $X$ be the character group of $T$. Then we get an exact sequence

$$
0 \rightarrow T \rightarrow T_{\log } \rightarrow \mathcal{H} \operatorname{om}_{S_{\mathrm{kfl}}}\left(X, \mathbb{G}_{\mathrm{m}, \log } / \mathbb{G}_{\mathrm{m}}\right) \rightarrow 0
$$

Because $\mathbb{G}_{\mathrm{m}, \log } / \mathbb{G}_{\mathrm{m}}$ is torsion free, we get $T[n]=T_{\log }[n]$. Then we get a short exact sequence $0 \rightarrow$ $T[n] \rightarrow G_{\log }[n] \rightarrow B[n] \rightarrow 0$. Therefore, $G_{\log }[n] \in(\mathrm{fin} / S)_{\mathrm{r}}$ by Proposition 5.1. Applying Proposition 5.1 again to the short exact sequence

$$
0 \rightarrow G_{\log }[n] \rightarrow T_{\mathbb{Z} / n \mathbb{Z}}\left(M^{\log }\right) \rightarrow Y / n Y \rightarrow 0,
$$

we get $T_{\mathbb{Z} / n \mathbb{Z}}\left(M^{\log }\right) \in(\text { fin } / S)_{\mathrm{r}}$.

Part (3) is clearly true for the two két $\log 1$-motives $[Y \rightarrow 0]$ and $\left[0 \rightarrow G_{\log }\right]$. It follows that it also holds for $M^{\log }$. 
At last, we prove part (4). By the proof of part (2) we get $T_{\mathbb{Z} / n \mathbb{Z}}\left(M^{\log }\right) \in(\mathrm{fin} / S)_{\mathrm{r}}$. Similarly, we have $T_{\mathbb{Z} / n \mathbb{Z}}\left(M^{\log }\right)^{*}=T_{\mathbb{Z} / n \mathbb{Z}}\left(\left(M^{\log }\right)^{\vee}\right) \in(\mathrm{fin} / S)_{\mathrm{r}}$, where $\left(M^{\log }\right)^{\vee}$ denotes the dual of the log 1-motive $M^{\log }$. It follows that $T_{\mathbb{Z} / n \mathbb{Z}}\left(M^{\log }\right) \in(\text { fin } / S)_{\mathrm{d}}$.

Definition 5.4. Let $S$ be a locally noetherian fs log scheme,

$$
M^{\log }=\left[Y \stackrel{u}{\rightarrow} G_{\log }\right]
$$

a két $\log 1$-motive over $S$ and $p$ a prime number. The két $\log p$-divisible group of $M^{\log }$ is defined to be $M^{\log }\left[p^{\infty}\right]:=\bigcup_{n} T_{\mathbb{Z} / p^{n} \mathbb{Z}}\left(M^{\log }\right)$.

\subsection{Extending finite group schemes associated to tamely ramified strict 1-motives}

Theorem 5.1. Let the notation and the assumptions be as in Theorem 3.1, and let $n$ be a positive integer.

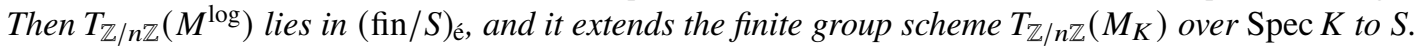

Proof. Because $T_{\mathbb{Z} / n \mathbb{Z}}\left(M^{\log }\right) \times_{S} S^{\prime}=T_{\mathbb{Z} / n \mathbb{Z}}\left(M^{\log } \times_{S} S^{\prime}\right) \in(\text { fin } / S)_{\mathrm{r}}$ and $S^{\prime}$ is a Kummer étale cover of

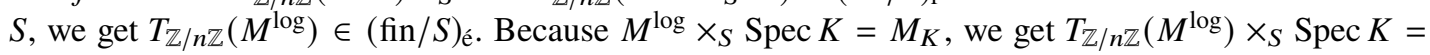
$T_{\mathbb{Z} / n \mathbb{Z}}\left(M_{K}\right)$.

The following theorem is stated in [12, §4.3] without proof. Here we present a proof.

Theorem 5.2 (Kato). Let $K$ be a complete discrete valuation field with ring of integers $R$, $p$ a prime number and $A_{K}$ a tamely ramified abelian variety over $K$. We endow $S:=\operatorname{Spec} R$ with the canonical log structure. Then the $p$-divisible group $A_{K}\left[p^{\infty}\right]$ of $A_{K}$ extends to an object of ( $p$-div $\left./ S\right)_{e}^{\log }$. It extends to an object of $(p-\operatorname{div} / S)_{\mathrm{d}}^{\log }$ if any of the following two conditions is satisfied:

(1) $A_{K}$ has semistable reduction.

(2) $p$ is invertible in $R$.

Proof. By $[16, \S 4.2]$, there exists a tamely ramified strict 1-motive

$$
M_{K}=\left[Y_{K} \stackrel{u_{K}}{\longrightarrow} G_{K}\right]
$$

such that $M_{K}\left[p^{\infty}\right]=A_{K}\left[p^{\infty}\right]$, and both $Y_{K}$ and $G_{K}$ have good reduction provided that $A_{K}$ has semistable reduction. By Theorem 3.1, $M_{K}$ extends to a két $\log 1$-motive $M^{\log }=\left[Y \stackrel{u^{\log }}{\longrightarrow} G_{\log }\right]$. Then $M_{K}\left[p^{\infty}\right]$ extends to $M^{\log }\left[p^{\infty}\right] \in(p-\operatorname{div} / S)_{\text {é }}^{\log }$ by Theorem 5.1 .

If $A_{K}$ has semistable reduction, then both $Y_{K}$ and $G_{K}$ have good reduction. Therefore, the két log 1-motive $M^{\log }$ is actually a log 1-motive over $S$ by Corollary 3.1. It follows that $M^{\log }\left[p^{\infty}\right] \in(p \text {-div/S })_{\mathrm{d}}^{\log }$.

If $p$ is invertible in $R$, then the object $T_{\mathbb{Z} / p^{n} \mathbb{Z}}\left(M^{\log }\right) \in(\text { fin } / S)_{\text {é }}$ actually lies in (fin/S $)_{\mathrm{d}}$ by [12, Prop. 2.1]. It follows that $M^{\log }\left[p^{\infty}\right] \in(p-\operatorname{div} / S)_{\mathrm{d}}^{\log }$.

Acknowledgments. In an email, Professor Chikara Nakayama informed the author that Professor Kazuya Kato thought it plausible that every abelian variety (not necessarily with semistable reduction) over a complete discrete valuation field extends uniquely to a Kummer log flat log abelian variety over the corresponding discrete valuation ring. This work is partly motivated by that piece of information. It is also motivated by Theorem 5.2, which is taken from [12, §4.3]. The author thanks Professor Chikara Nakayama for his generosity. The author also thanks Professor Ulrich Görtz for very helpful discussions concerning taking quotient for equivalence relations, as well as for his support during the past few years.

The author thanks the anonymous referee for her or his corrections. More important, he thanks the referee for her or his questions and comments that not just motivated the author to give a more systematic treatment to the results in Section 3 but also directed him to possible research problems in the future.

This work has been partially supported by SFB/TR 45 'Periods, Moduli Spaces and Arithmetic of Algebraic Varieties'. 


\section{References}

[1] A. Bertapelle, M. Candilera and V. Cristante, 'Monodromy of logarithmic Barsotti-Tate groups attached to 1-motives', J. Reine Angew. Math. 573 (2004), 211-234.

[2] S. Bosch, W. Lütkebohmert and M. Raynaud, Néron Models, volume 21 of Ergebnisse der Mathematik und ihrer Grenzgebiete (3) [Results in Mathematics and Related Areas (3)] (Springer-Verlag, Berlin, 1990).

[3] P. Deligne, 'Théorie de Hodge. III', Publ. Math. Inst. Hautes Études Sci. 44 (1974), 5-77.

[4] P. Deligne, 'Le lemme de gabber', Astérisque 127(5) (1985), 131-150.

[5] A. Grothendieck, M. Raynaud and D. S. Rim, Groupes de monodromie en géométrie algébrique. I Monodromy groups in algebraic geometry. I, Vol. 288 of Lecture Notes in Mathematics (Springer-Verlag, Berlin-New York, 1972). Séminaire de Géométrie Algébrique du Bois-Marie 1967-1969 (SGA 7 I).

[6] L. H. Halle and J. Nicaise, Néron Models and Base Change, Vol. 2156 of Lecture Notes in Mathematics (Springer, Cham, Switzerland, 2016).

[7] L. Illusie, 'An overview of the work of K. Fujiwara, K. Kato, and C. Nakayama on logarithmic étale cohomology. Cohomologies $p$-adiques et applications arithmétiques, II'p-adic cohomologies and arithmetic applications. II, Astérisque 279 (2002), 271-322.

[8] T. Kajiwara, K. Kato and C. Nakayama, 'Logarithmic abelian varieties', Nagoya Math. J. 189 (2008), $63-138$.

[9] T. Kajiwara, K. Kato and C. Nakayama, 'Logarithmic abelian varieties, part IV: proper models', Nagoya Math. J. 219 (2015), 9-63.

[10] T. Kajiwara, K. Kato and C. Nakayama, 'Logarithmic abelian varieties, part VI: local moduli and GAGF', Yokohama Math. J. 65 (2019), 53-75.

[11] K. Kato, 'Logarithmic structures of Fontaine-Illusie. II', Preprint, 2019, arXiv:1905.10678.

[12] K. Kato, Logarithmic Dieudonné Theory, Preprint, 1992.

[13] K. Kato and F. Trihan, 'On the conjectures of Birch and Swinnerton-Dyer in characteristic $p>0$ ', Invent. Math. 153(3) (2003), 537-592.

[14] K. Madapusi Sampath, Log p-divisible groups (D’aprés Kato) (2009). URL: https://sites.google.com/a/bc.edu/keerthi/.

[15] W. Nizioł, ' $K$-theory of log-schemes. I', Doc. Math. 13 (2008), 505-551.

[16] M. Raynaud, '1-motifs et monodromie géométrique’ 1-motives and geometric monodromy, Astérisque 223 (1994), 295 -319.

[17] The Stacks Project Authors, Stacks Project (2020). URL: http://stacks.math.columbia.edu.

[18] J. Stix, 'Projective anabelian curves in positive characteristic and descent theory for log-étale covers', Bonner Mathematische Schriften 354 (2002).

[19] H. Zhao, 'Log abelian varieties over a log point', Doc. Math. 22 (2017), 505-550. 